\title{
Zonal-Mean Atmospheric Dynamics of Slowly Rotating Terrestrial Planets
}

\author{
G. J. COLYER AND G. K. VALLIS \\ College of Engineering, Mathematics and Physical Sciences, University of Exeter, Exeter, United Kingdom
}

(Manuscript received 26 June 2018, in final form 23 January 2019)

\begin{abstract}
The zonal-mean atmospheric flow of an idealized terrestrial planet is investigated using both numerical simulations and zonally symmetric theories, focusing largely on the limit of low planetary rotation rate. Two versions of a zonally symmetric theory are considered, the standard Held-Hou model, which features a discontinuous zonal wind at the edge of the Hadley cell, and a variant with continuous zonal wind but discontinuous temperature. The two models have different scalings for the boundary latitude and zonal wind. Numerical simulations are found to have smoother temperature profiles than either model, with no temperature or velocity discontinuities even in zonally symmetric simulations. Continuity is achieved in part by the presence of an overturning circulation poleward of the point of maximum zonal wind, which allows the zonal velocity profile to be smoother than the original theory without the temperature discontinuities of the variant theory. Zonally symmetric simulations generally fall between the two sets of theoretical scalings, and have a faster polar zonal flow than either set. Three-dimensional simulations, which allow for the eddy motion that is missing from both models, fall closer to the scalings of the variant model. At very low rotation rates the maximum zonal wind falls with falling planetary rotation rate, and is zero at zero rotation. The low-rotation limit of the overturning circulation, however, is strong enough to drive the temperature profile close to a state of nearly constant potential temperature.
\end{abstract}

\section{Introduction}

The theory of the Hadley cell has long been an object of study. Of both historical and scientific note is the famous paper by Hadley on the trade winds over 250 years ago (Hadley 1735), and the work a century or so later by Ferrel (1859) and Thomson (1892). Ferrel introduced the notion of a second cell (now called the Ferrel cell), but none of these authors were able to give a proper explanation of the limited latitudinal extent of the Hadley cell, which they generally envisioned to extend to the pole. Baroclinic instability was implicitly considered to be a limiting factor in the Hadley cell extent in the discussion of Lorenz (1967), but even without that instability an ideal Hadley cell cannot extend to the pole. The reason for that comes from the conservation of angular momentum in the poleward-moving branch of the Hadley cell, as noted by Schneider (1977), which in the absence of frictional effects leads to the development of very strong zonal winds. Noting that result, Held and Hou (1980,

Corresponding author address: G. J. Colyer, Harrison Building, North Park Road, Exeter, EX4 4QF, United Kingdom. hereafter $\mathrm{HH}$ ) developed a zonally symmetric theory in which, neglecting eddies and any time dependence, they posited a circulation at low latitudes in which the total zonal specific angular momentum is conserved by the flow, and a purely zonal flow at high latitudes in thermal wind balance with the specified forcing. Matching conditions are applied at the boundary between the low- and high-latitude regions, and the satisfaction of these determines the boundary latitude $\theta_{H}$. Their model may be regarded as a theory for an "ideal" axisymmetric Hadley circulation, and one of its main contributions was to show that even in the absence of baroclinic instability the Hadley cell would not reach the pole, at least on a rapidly rotating planet like Earth.

$\mathrm{HH}$ expressed their theory in fairly general terms, but focused on the limit $\theta_{H} \ll 1$, which corresponds to high planetary rotation rate $\Omega$. The low- $\Omega$ limit of the theory was then specifically considered by Hou (1984), with Covey et al. (1986) and Mitchell and Vallis (2010) performing a number of related simulations, looking at superrotation in particular and motivated in part by Titan. Various extensions to the theory relevant to Earth have also taken place. For example, Lindzen and Hou (1988) and Plumb and Hou (1992) considered hemispherically 
asymmetric forcing, and Caballero et al. (2008) extended the zonally symmetric theory, a Boussinesq model originally, to compressible atmospheres.

In this paper, motivated partly by Venus (which has an obliquity of only $3^{\circ}$ ), we revisit the hemispherically symmetric case, focusing on the matching conditions between the low- and high-latitude regions, and on the low-rotation regime. We also perform both zonally symmetric and three-dimensional simulations using an idealized GCM. Noting some of the differences between these simulations and the theory of $\mathrm{HH}$, we are motivated to evaluate an alternative matching condition in the zonally symmetric theory, which exchanges the discontinuity in zonal wind for one in temperature. We compare the theoretical predictions and obtain scalings for both theories in the low- $\Omega$ limit. In this limit, it is natural to use the boundary colatitude $\varphi_{H} \equiv \pi / 2-\theta_{H} \ll 1$. We go on to compare the simulations with both of the theories, and discuss how they may be reconciled, in particular in the polar region, and how this may be able to effect the elimination of both discontinuities. To facilitate comparisons between theory and simulation, we use only a simple forcing in the simulations, that of Held and Suarez (1994), which is very similar to that of HH. Both use Newtonian relaxation of the temperature field toward a specified equilibrium that is maximum at the equator and minimum at the poles, without diurnal or seasonal variation.

An outline of the paper follows. Section 2 contains a summary of the Held-Hou theory, including discussion of the matching conditions and the general solution. Section 3 introduces the alternative matching condition and its consequences. The two theories are compared further in section 4 , and then in section 5 the low- $\Omega$ limit is considered and various scalings obtained. Section 6 describes the numerical modeling and results, with comparison to theory and consequent discussion. In section 7 we draw attention to some implications for the idealized modeling of Venus, but we leave the actual study of Venus to a later paper. We conclude in section 8. An appendix provides more detail concerning the conversion used to compare the theory with the simulations.

\section{The Held-Hou theory for zonally symmetric atmospheres}

\section{a. Summary of derivation}

We first summarize the theory of HH. Readers who are familiar with it may wish to skip to section 3, referring back to this section as needed. HH start from the Boussinesq version of the hydrostatic primitive equations on a sphere. [The compressible hydrostatic primitive equations in pressure coordinates have the same form as the Boussinesq equations (Vallis 2017), so the Boussinesq approximation is not as restrictive as it may seem.] Steady flow $(\partial / \partial t=0$, where $t$ is time $)$ and zonal symmetry $(\partial / \partial \phi=0$, where $\phi$ is longitude) are assumed throughout, and we will only use the equations in their inviscid approximation. The two horizontal components of the momentum equation are

$$
\begin{aligned}
& \frac{D u}{D t}-f v-\frac{u v \tan \theta}{a}=0, \\
& \frac{D v}{D t}+f u+\frac{u^{2} \tan \theta}{a}=-\frac{1}{a} \frac{\partial \Phi}{\partial \theta},
\end{aligned}
$$

where $\theta$ is latitude, $z$ is height above the surface, $a$ is the planetary radius, $\mathbf{v}(\theta, z)=(u, v, w)$ is the flow velocity, $f=2 \Omega \sin \theta$ is the Coriolis parameter, $\Phi$ is the geopotential, and $D / D t=(v / a) \partial / \partial \theta+w \partial / \partial z$. The equations are completed by the incompressibility condition

$$
\nabla \cdot \mathbf{v}=\frac{1}{a \cos \theta} \frac{\partial(v \cos \theta)}{\partial \theta}+\frac{\partial w}{\partial z}=0,
$$

hydrostasy

$$
\frac{\partial \Phi}{\partial z}=\frac{g \Theta}{\Theta_{0}},
$$

where $g$ is the acceleration due to gravity and $\Theta$ is potential temperature, and the thermodynamic equation

$$
\frac{D \Theta}{D t}=-\frac{\left(\Theta-\Theta_{E}\right)}{\tau} .
$$

Radiative-convective equilibration is represented by the thermal forcing term on the right-hand side that relaxes the potential temperature toward a specified equilibrium $\Theta_{E}$ [see (12) below] with a specified time $\tau$.

At the top of the atmosphere $z=H$ we take $w=0$, and (1) may then be written

$$
v(\zeta+f)=0,
$$

where

$$
\zeta=-\frac{1}{a} \frac{\partial u}{\partial \theta}+\frac{u \tan \theta}{a}
$$

is (the vertical component of) the relative vorticity. Equation (6) may be satisfied in two ways: (i) $v=0$ (identically), which by (3) implies $w=0$, self-consistently; we will call $\mathbf{v}=(u, 0,0)$ a circulation-free solution; or (ii) total vorticity $\zeta+f=0$, that is,

$$
-\frac{1}{a} \frac{\partial u}{\partial \theta}+f+\frac{u \tan \theta}{a}=-\frac{1}{a^{2} \cos \theta} \frac{\partial M}{\partial \theta}=0,
$$


conserving the total zonal specific angular momentum $M \equiv(u+\Omega a \cos \theta) a \cos \theta$; this has solution

$$
u=u_{M}(\theta)+\frac{u(0)}{\cos \theta},
$$

where

$$
u_{M} \equiv \frac{\Omega a \sin ^{2} \theta}{\cos \theta} .
$$

The alternatives (i) and (ii) will be applied to high- and low-latitude regions respectively [note that (10) would diverge at the pole], with matching conditions at the boundary latitude $\theta_{H}$, which is the edge of the Hadley cell. [In some monsoon contexts the alternatives may both apply at low latitudes but in different regimes, as in Geen et al. (2018).]

The remaining dynamical equations may be combined by cross differentiating-assuming that the advective term $\mathbf{v} \cdot \nabla v$ in (2), which is zero in case (i), is also small in case (ii) - to produce an equation for thermal gradient wind balance, and integrating in $z$-assuming that $u$ is small in the boundary layer-to get at $z=H$ :

$$
f u+\frac{u^{2} \tan \theta}{a}=-\frac{g H}{a \Theta_{0}} \frac{\partial \bar{\Theta}}{\partial \theta},
$$

where the overbar denotes the vertical average $H^{-1} \int_{0}^{H} d z$. The first and second terms on the left-hand side correspond to the geostrophic and cyclostrophic thermal wind gradients respectively.

In the circulation-free case (i), we now use the thermodynamic equation, (5), to conclude that $\Theta=\Theta_{E}$. Equation (11) must then be solved for $u \equiv u_{E}$ given $\overline{\boldsymbol{\Theta}}=\overline{\boldsymbol{\Theta}}_{E}$, which is specified as

$$
\frac{\bar{\Theta}_{E}}{\Theta_{0}}=1-\frac{2}{3} \Delta_{H} P_{2}(\sin \theta),
$$

where $P_{2}(x)=\left(3 x^{2}-1\right) / 2$ and $\Theta_{0}$ and $\Delta_{H}$ are parameters. The solution is

$$
u_{E}=\Omega a \cos \theta(\sqrt{2 R+1}-1),
$$

where $R \equiv \Delta_{H} g H / \Omega^{2} a^{2}$, as in HH. We note that this $u_{E}$ corresponds to global rigid-body rotation about the planetary axis, that is, constant angular velocity $u_{E} / a \cos \theta=\sqrt{2 R+1}-1$. This is a consequence of the specified form of (12).

In the angular-momentum-conserving case (ii), $u$ is given by (9), and (11) becomes an equation to be solved for $\bar{\Theta}$. Equivalently, in this case (11) may be written in the form of a conserved energy: first multiply (8) by $u$ to get

$$
\frac{1}{2 a} \frac{\partial}{\partial \theta}\left(u^{2}\right)=f u+\frac{u^{2} \tan \theta}{a},
$$

and hence

$$
\frac{\partial}{\partial \theta}\left(\frac{1}{2} u^{2}+\frac{g H \bar{\Theta}}{\Theta_{0}}\right)=0
$$

which consists of a zonal kinetic energy term and a thermal term. The solution is

$$
\frac{\bar{\Theta}(0)-\bar{\Theta}}{\Theta_{0}}=\frac{u^{2}-u(0)^{2}}{2 g H} .
$$

This immediately implies a finite upper bound on $u$, since $\bar{\Theta}>0$. Specializing to the case $u(0)=0,{ }^{1}(16)$ becomes

$$
\frac{\bar{\Theta}(0)-\bar{\Theta}}{\Theta_{0}}=\frac{u_{M}^{2}}{2 g H}=\frac{\Omega^{2} a^{2} \sin ^{4} \theta}{2 g H \cos ^{2} \theta},
$$

which is (12) of $\mathrm{HH}$.

\section{b. Matching conditions}

$\mathrm{HH}$ proposed the following two matching conditions: continuity of temperature,

$$
\bar{\Theta}\left(\theta_{H}-\right)=\bar{\Theta}_{E}\left(\theta_{H}+\right),
$$

and the closure of the energy budget over the Hadley cell, integrating (5) and using $\mathbf{v} \cdot \nabla \Theta=\nabla \cdot(\mathbf{v} \Theta)$, implied by (3), to obtain

$$
\int_{0}^{\theta_{H}} \bar{\Theta} \cos \theta d \theta=\int_{0}^{\theta_{H}} \bar{\Theta}_{E} \cos \theta d \theta
$$

Since $\Theta=\Theta_{E}$ in the high-latitude region, this region may be added to the integral to write

$$
\int_{0}^{\pi / 2}\left(\bar{\Theta}-\bar{\Theta}_{E}\right) \cos \theta d \theta=0
$$

The second matching condition implies $\bar{\Theta} \sim \bar{\Theta}_{E} \sim \Theta_{0}$ and hence that the upper bound on $u \sim \sqrt{g H}$.

\section{c. General solution}

HH focused mainly on the low- $R$ limit, and we will focus mainly on the high- $R$ limit considered by Hou (1984). However, we first describe the general solution.

\footnotetext{
${ }^{1}$ This eliminates both equatorial superrotation, $u(0)>0$, in accordance with Hide (1969), and nonequatorial extrema in $\bar{\Theta}$, which occur where $u=0$ if $u(0)<0$.
} 
The zonal wind $u$ is already given on either side of $\theta_{H}$ by (10) and (13), but $\theta_{H}$ is still to be determined; $\bar{\Theta}$ is given on either side of $\theta_{H}$ by (12) and (17), in which the only other unknown is $\bar{\Theta}(0)$. These may be substituted into the second matching condition (19) which upon integration gives

$$
\begin{aligned}
\frac{\bar{\Theta}(0)}{\Theta_{0}}= & 1+\Delta_{H}\left[\frac{1}{3}-\frac{1}{3} x_{H}^{2}\left(1+\frac{1}{2 R}\right)-\frac{1}{2 R}\right. \\
& \left.+\frac{1}{4 R x_{H}} \ln \left(\frac{1+x_{H}}{1-x_{H}}\right)\right],
\end{aligned}
$$

where $x_{H} \equiv \sin \theta_{H}$. The first matching condition may be used to eliminate $\bar{\Theta}(0)$ and obtain the expression

$$
R=\frac{3}{4}\left[\frac{1}{3}+\frac{1}{x_{H}^{2}}+\frac{x_{H}^{2}}{1-x_{H}^{2}}-\frac{1}{2 x_{H}^{3}} \ln \left(\frac{1+x_{H}}{1-x_{H}}\right)\right],
$$

which is implicit in (17) of $\mathrm{HH}$ and may be inverted numerically, that is, solved for $x_{H}$ given $R . \bar{\Theta}(0)$ and hence $\bar{\Theta}$ generally is then determined.

Figure 1 shows a sketch of the solutions for (Fig. 1a) $u$ and (Fig. 1b) $\bar{\Theta}$, and how they vary with $\Omega$. The dashdotted lines show $u_{E}$ and $\bar{\Theta}_{E}$ respectively. For each $\Omega$ (each color), the solid lines show the angular-momentumconserving solutions (which both diverge at the pole), the thick lines are the combined $\mathrm{HH}$ solutions, and the vertical dashed lines mark $\theta_{H}$-where the temperature curves cross, by the first matching condition. The second matching condition sets the equatorial intercept of temperature such that the areas under the $\bar{\Theta}$ and $\bar{\Theta}_{E}$ curves are equal, or equivalently that the net area between the curves is zero, when weighted by $\cos \theta$. [Note however that this $\cos \theta$ weighting factor, from (19) or (20), is not incorporated into the sketched curves. It has the same functional form as $u_{E}$.] A notable feature of the $\mathrm{HH}$ theory is that $u$ is discontinuous at $\theta_{H}$. This corresponds, via thermal wind balance, to the discontinuous gradient in $\bar{\Theta}$, even though $\bar{\Theta}$ itself is continuous.

As $\Omega$ decreases (red then black then blue), both $u$ and $\bar{\Theta}$ flatten in the Hadley cell region, by (10) and (17) respectively, and $\theta_{H}$ moves poleward. To preserve the equal-area constraint, the low-latitude temperature is reduced. Although $u_{M}$ is also reduced at any given $\theta$, the discontinuity in $u$ at $\theta_{H}$ is increased. This fact, in part, motivates us to suggest an alternative matching condition, which we consider in the next section. In section 5 we will develop the low- $\Omega$ scalings of the two theories quantitatively.

Finally we note that, at the boundary $x_{H}$, the ratio of gradients $u_{M}^{\prime} / u_{E}^{\prime}$ diverges $\sim-2 / R$ as $R \rightarrow 0$; both $u_{M}^{\prime} / u_{M}^{\prime}$ and $u_{M} / u_{E}$ diverge $\sim-2 \sqrt{2 R} / 3$ as $R \rightarrow \infty$. (a)

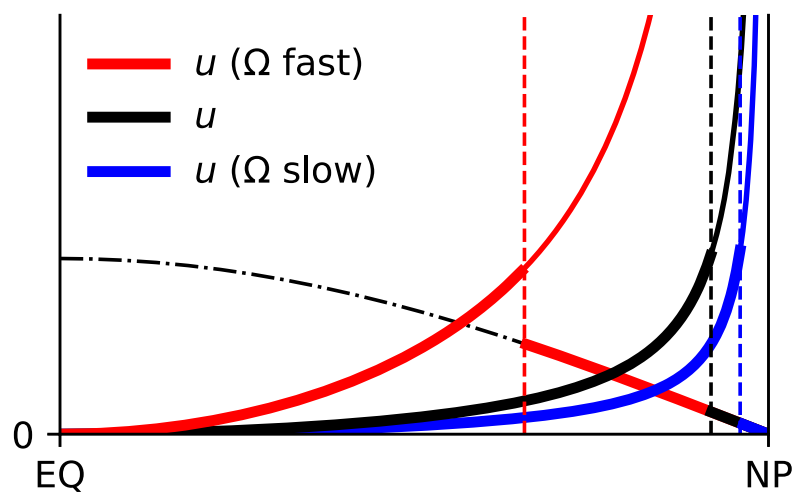

(b)

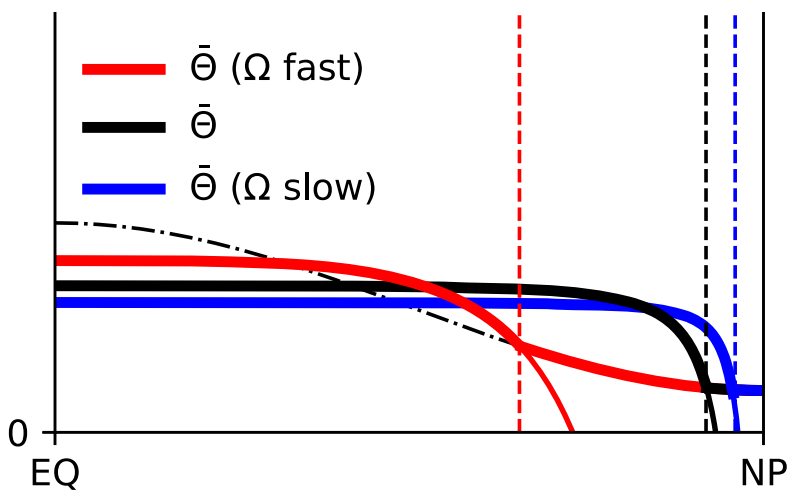

FIG. 1. Sketch of the Held-Hou model (cf. Figs. 1 and 3 of HH) for three different planetary rotation rates $\Omega$, indicated by different colors. (a) Zonal wind vs latitude. The solid line shows the angularmomentum-conserving zonal wind $u_{M}$, which diverges at the pole. The dash-dotted line shows the zonal wind $u_{E}$ that is in thermal wind balance with $\bar{\Theta}_{E}$. (b) Potential temperature. The solid line shows the temperature $\bar{\Theta}$ in thermal wind balance with $u_{M}$; this too diverges at the pole. The dash-dotted line shows the forcing temperature $\bar{\Theta}_{E}$. The original $\mathrm{HH}$ matching conditions determine the latitude (vertical dashed lines in both panels) at which one solution crosses over to the other, where $\bar{\Theta}$ is continuous but $u$ is discontinuous (thick solid lines).

\section{An alternative matching condition}

We will see below that the discontinuity in $u$ at $\theta_{H}$ (shown as the red curve in Fig. 3b) is of the same order as $u$ itself (on the equatorward side of higher $u$-and also, in the low-rotation limit, is much larger than $u$ on the poleward side). We will also see (in section 6) that our numerical simulations of a primitive equation model, even in the zonally symmetric case and in the inviscid limit, do not seem to reach a steady-state velocity profile with a strong discontinuity. This is associated with both temporal fluctuations and a weak overturning circulation on the poleward side of the maximum $u$. However, we first consider an alternative theoretical model that would allow us to avoid the 
zonal-wind discontinuity without relaxing the assumptions made in either the equatorward or the poleward region. Namely, we could change the first matching condition to specify that $u$ itself be continuous at the boundary latitude:

$$
u_{M}\left(\theta_{H}^{-}\right)=u_{E}\left(\theta_{H}+\right) .
$$

Using (10) and (13), this gives the boundary latitude directly:

$$
\cos ^{2} \theta_{H}=1-x_{H}^{2}=\frac{1}{\sqrt{2 R+1}} .
$$

These two equations replace (18) and (22). Equations (19) and (20) are essentially a consequence of the dynamics, and have not been changed; (21) was deliberately written in a form that is independent of the first matching condition, so $\bar{\Theta}(0)$ is determined as before. The consequence, unsurprisingly, is that there is now a discontinuity in $\bar{\Theta}$ instead of $u$. We will return to discuss this trade-off later.

The significance of the crossover point between the $u_{M}$ and $u_{E}$ curves was already pointed out by Held and Hoskins (1985). In a simple model similar to that of $\mathrm{HH}$, but with a linear damping term retained in (6), they show that $u \leq u_{M}$, and therefore the Hadley cell must extend from the equator at least as far as this crossover point before it can switch to the $u_{E}$ branch. Thus, our alternative matching condition here corresponds to attaining this lower bound on the crossover latitude; the extension beyond this bound in the original $\mathrm{HH}$ theory has a different physical origin, captured by their matching condition, which may be differently affected by any modifications to the poleward region.

Figure 2 shows a sketch similar to Fig. 1 but for the new matching condition. The underlying $u_{M}$ and $u_{E}$ curves are all as before, but now the boundary latitude is set by where they-and not the temperature curves-cross. The boundary still moves poleward as $\Omega$ is decreased, but for all values of $\Omega$ the boundary is equatorward of the original boundary. The temperature discontinuity at the new boundary is evident. It is given by adding (12) to (17), using (21), and (24) to eliminate $R$ :

$$
\begin{aligned}
\frac{\bar{\Theta}\left(x_{H}-\right)-\bar{\Theta}_{E}\left(x_{H}+\right)}{\Theta_{0}}= & \frac{\Delta_{H}}{\left(2-x_{H}^{2}\right)}\left[\frac{5}{3}-\frac{1}{x_{H}^{2}}\right. \\
& \left.+\frac{\left(1-x_{H}^{2}\right)^{2}}{2 x_{H}^{3}} \ln \left(\frac{1+x_{H}}{1-x_{H}}\right)\right] .
\end{aligned}
$$

Again the discontinuity (but now in temperature) becomes larger as $\Omega$ is reduced. The temperature gradients (a)

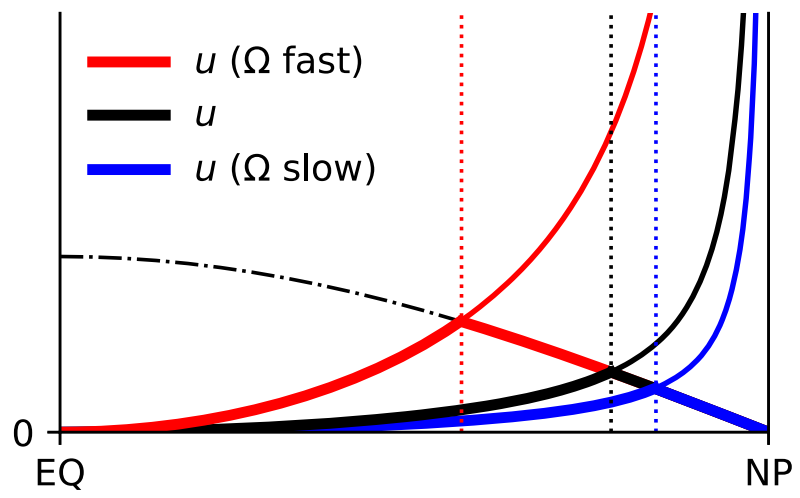

(b)

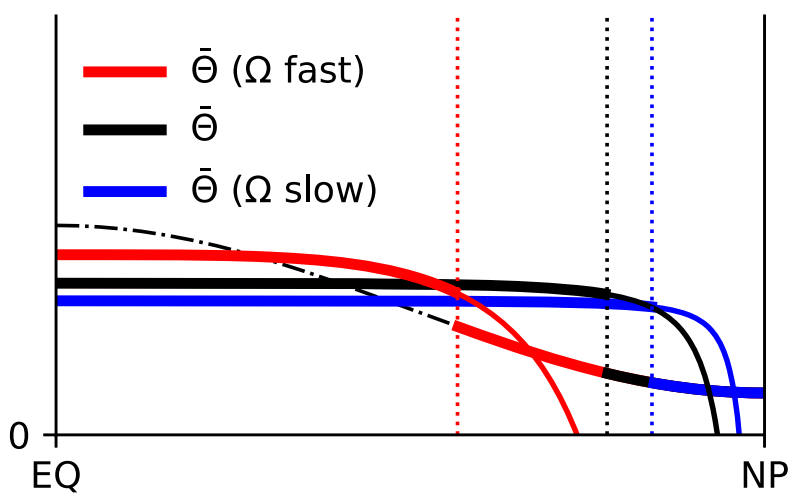

FIG. 2. As in Fig. 1, but for the model with modified matching conditions that determine the latitude (vertical dotted lines in both panels) at which one solution crosses over to the other, where $u$ is continuous but $\bar{\Theta}$ is not (thick solid lines).

on either side of the discontinuity are equal, by thermal wind balance. In order still to satisfy the second matching condition with the new boundary, the equatorial temperature is shifted slightly higher (relative to Fig. 1, for a given $\Omega$ ), to maintain the equal areas. However, this shift is always small (and actually vanishes in both the low- and high-rotation limits), as seen in Fig. 3e below.

With the new matching condition, the ratio of $u$ gradients at the boundary is

$$
\left.\frac{u_{M}^{\prime}}{u_{M}^{\prime}}\right|_{x_{H}}=-\left(\frac{\sqrt{2 R+1}+1}{\sqrt{2 R+1}-1}\right),
$$

which has the limit -1 (unlike the divergence with the original matching condition) as $R \rightarrow \infty$; it still diverges $\sim-2 / R$ as $R \rightarrow 0$.

\section{Comparison of the two variants}

Figure 3 shows a quantitative comparison of various other aspects of the two theories. Note that at high $\Omega$ 
(a)

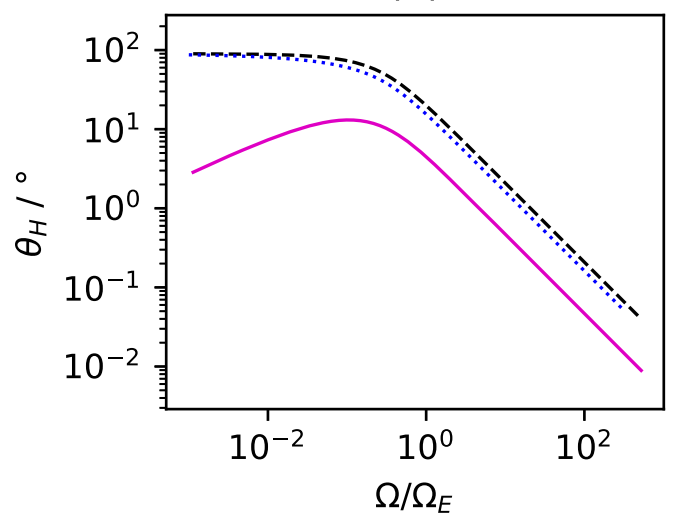

(c)

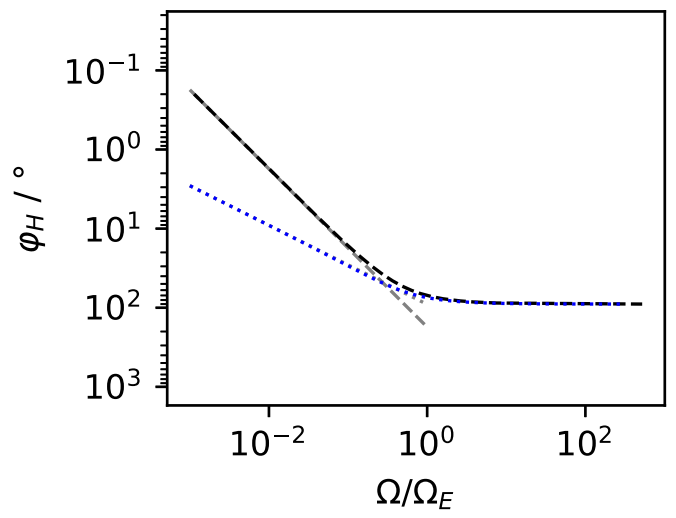

(e)

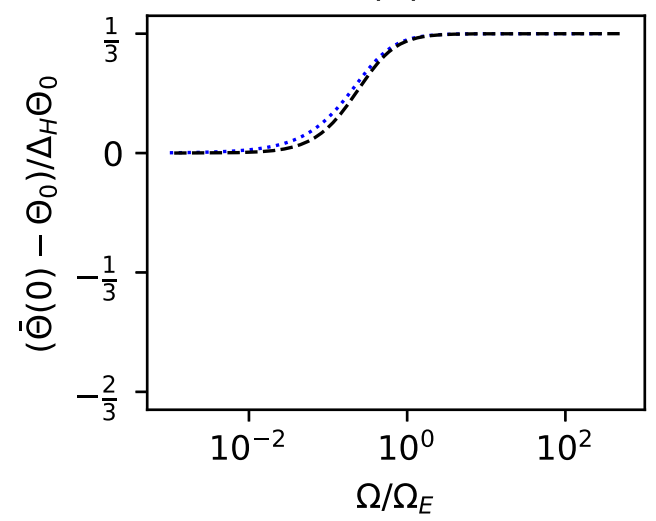

(b)

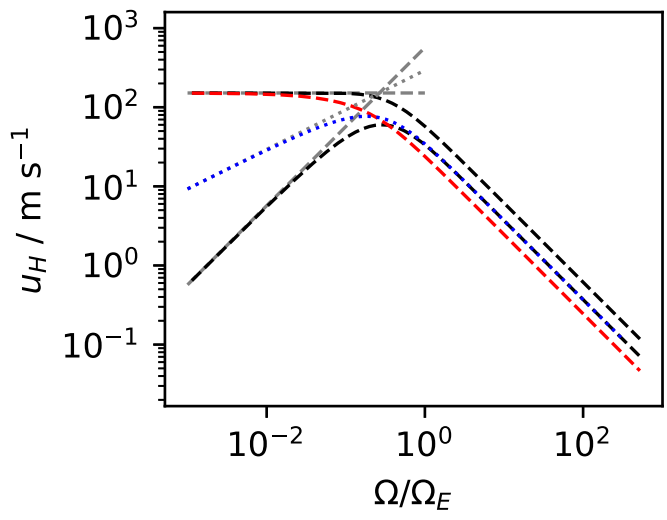

(d)

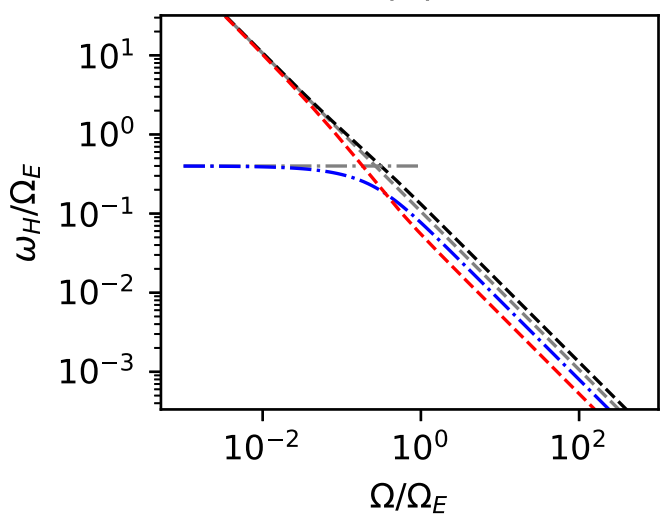

(f)

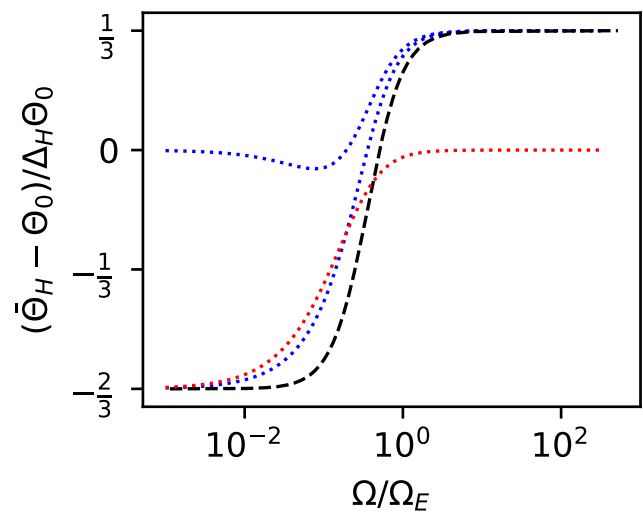

FIG. 3. Theoretical solutions of the HH and continuous- $u$ theories. Parameters appropriate for Earth have been used, so that these plots match Fig. 7, and the variable planetary rotation rate $\Omega$ has been normalized to Earth's rotation rate $\Omega_{E}$. In all panels, dashed lines correspond to the HH theory, dotted lines to the continuous- $u$ theory, and dash-dotted lines to both. (a) Hadley cell boundary latitude $\theta_{H}$, (b) boundary zonal wind $u_{H}$, (c) boundary colatitude $\varphi_{H} \equiv \pi / 2-\theta_{H}$, (d) boundary zonal wind as an angular velocity around the planetary axis $\omega_{H} \equiv$ $u_{H} /\left(a \cos \theta_{H}\right)$, (e) equatorial temperature $\bar{\Theta}(0)$, and (f) boundary temperature $\bar{\Theta}_{H}$. The red curves in (b), (d), and (f) show the discontinuity (difference) at the boundary, between the appropriate pair. The solid magenta curve in (a) shows the difference between the two theories. Gray straight lines indicate the low- $\Omega$ asymptotes derived in the text and also shown in Fig. 7. 
(low $R$ ), which is the limit originally considered by $\mathrm{HH}$, all of the power-law scalings shown are the same for both theories; they differ only by an order-unity prefactor. Furthermore, the boundary latitude $\theta_{H}$ is close across the whole range of $\Omega$ (differing by no more than $\sim 10^{\circ}$ ), as is the equatorial temperature $\bar{\Theta}(0)$. However, different scalings are obtained from the two theories at low $\Omega$ (high $R$ ), including for the boundary colatitude $\varphi_{H}$. The alternative boundary condition is therefore mainly of interest in this limit, in which it is natural to use the colatitude $\varphi \equiv \pi / 2-\theta$ instead of $\theta$. In the following section we derive the low- $\Omega$ scalings indicated by the gray lines in Fig. 3, and then in section 6 we compare them with numerical simulation results from a GCM code.

\section{The low-rotation limit and colatitude scalings}

\section{a. General}

We now turn to the limit $\Omega \rightarrow 0$. In the Hadley cell we see immediately from (17) that, except near the poles,

$$
\frac{\bar{\Theta}(0)-\bar{\Theta}}{\Theta_{0}}=\frac{\Omega^{2} a^{2} \sin ^{4} \theta}{2 g H \cos ^{2} \theta} \rightarrow 0,
$$

and so $\bar{\Theta} \rightarrow \bar{\Theta}(0)=$ const. This result was pointed out by Hou (1984). As mentioned above, it means that the temperature is flattened (relative to the forcing temperature) within the Hadley cell. This flattening is illustrated in Figs. 1 and 2. Toward the (north) pole it is natural to make the small-angle approximation for the colatitude $\varphi \equiv \pi / 2-\theta$, whence

$$
\frac{\bar{\Theta}(0)-\bar{\Theta}}{\Theta_{0}} \sim \frac{\Omega^{2} a^{2}}{2 g H \varphi^{2}} .
$$

For the circulation-free solution at the pole itself, (12) gives

$$
\frac{\bar{\Theta}_{E}}{\Theta_{0}}=1-\frac{2}{3} \Delta_{H}+\Delta_{H} \varphi^{2}+O\left(\varphi^{4}\right),
$$

which again is constant to leading order, and the second matching condition becomes simply

$$
\bar{\Theta}(0)=\Theta_{0} .
$$

From (10), the zonal wind toward the poleward edge of the Hadley cell is

$$
u_{M}(\varphi) \sim \frac{\Omega a}{\varphi} .
$$

On the poleward side of the boundary, we have cyclostrophic balance; taking the large- $R$ limit of (13),

$$
u_{E}(\varphi)=\sqrt{2 \Delta_{H} g H} \varphi .
$$

That $u_{E} \propto \varphi$ in this limit means the polar vortex rotates like a rigid body with angular velocity

$$
\frac{u_{E}}{a \varphi}=\frac{\sqrt{2 \Delta_{H} g H}}{a},
$$

which is independent of $\Omega$ (at fixed $\Delta_{H} g H$-not at fixed $R$ ). We noted the rigid-body rotation for this global $\bar{\Theta}_{E}$ earlier. In the present limit it depends only on the vanishing $\varphi$ and nonvanishing $\varphi^{2}$ terms in the polar expansion, (28). The polar vorticity (both relative and absolute) is $2 \sqrt{2 \Delta_{H} g H} / a$ in this limit.

\section{b. Low-rotation solutions with original $\mathrm{HH}$ matching conditions}

We impose (18) to determine the transition colatitude $\varphi_{H} \equiv \pi / 2-\theta_{H}$. Using (27), (28), and (29) we get, to leading order,

$$
1-\frac{\Omega^{2} a^{2}}{2 g H \varphi_{H}^{2}}=1-\frac{2}{3} \Delta_{H}
$$

and hence

$$
\varphi_{H}=\frac{\sqrt{3} \Omega a}{2 \sqrt{\Delta_{H} g H}}=\sqrt{\frac{3}{4 R}} .
$$

This last result was also given implicitly by Hou (1984) as part of his (34).

From (30), the maximum zonal wind, at the poleward edge of the Hadley cell, is therefore

$$
u_{M}\left(\varphi_{H}\right)=\frac{\Omega a}{\varphi_{H}}=\frac{2 \sqrt{\Delta_{H} g H}}{\sqrt{3}},
$$

which like the polar vorticity is independent of $\Omega$, as noted by Covey et al. (1986), and is consistent with the form of the upper bound on $u$ mentioned earlier. At the edge of the polar vortex, from (31),

$$
u_{E}\left(\varphi_{H}\right)=\sqrt{2 \Delta_{H} g H} \varphi_{H}=\sqrt{\frac{3}{2}} \Omega a .
$$

Thus, the Hadley cell extends with constant $\bar{\Theta}$ almost to the pole; at its edge, the maximum zonal wind is independent of $\Omega$, and beyond there is a polar vortex with rigid-body rotation whose angular velocity is independent of $\Omega$.

The dimensionless parameter $R$ is similar to a thermal Rossby number, at least in the limit $R \ll 1$, which corresponds to high $\Omega$ [and to the geostrophic limit of (11), 
in which only the first term appears on the left-hand side]. In this limit, $R \sim u_{E} / \Omega a$, and in the Hadley cell region $R \sim u_{M} / \Omega a$.

In the opposite limit here (the cyclostrophic limit), $R \gg 1$, and $\sqrt{R} \sim u_{M} / \Omega a$, whereas in the polar region $u_{E} / \Omega a$ is of order unity; in fact, using (35) and (36), in the cyclostrophic limit $u_{M}\left(\varphi_{H}\right) / u_{E}\left(\varphi_{H}\right)=2 \sqrt{2 R} / 3 \gg 1$. This is the large discontinuity in $u$ already discussed: of the same order as $u_{M}$, and in the low- $\Omega$ limit large compared to $u_{E}$ and $\Omega a$. Figure 1 is necessarily drawn for finite $\Omega$ but so as to be suggestive of this limit.

\section{c. Low-rotation solutions with continuous $u$}

In the low- $\Omega$ limit, (24) gives

$$
\varphi_{H}=\left(\frac{1}{2 R}\right)^{1 / 4}=\frac{\sqrt{\Omega a}}{\left(2 \Delta_{H} g H\right)^{1 / 4}},
$$

and hence, using (30) or (31),

$$
u\left(\varphi_{H}\right)=\sqrt{\Omega a}\left(2 \Delta_{H} g H\right)^{1 / 4} .
$$

The $\varphi_{H}$ given by (37), although larger than that given by (34), is still small; both approach the pole asymptotically as $\Omega \rightarrow 0$. In this limit, the closure integral, (20), is unaffected by the tiny polar region. We will return to this point when we discuss the numerical results below.

Note that the continuous- $u$ behavior circumvents a problematic issue in the original theory-namely that the maximum $u$, given by (35), remains nonzero even as $\Omega \rightarrow 0$. In the continuous- $u$ theory, the maximum $u$ is given by (38), and, although the polar vorticity stays constant, because the polar vortex shrinks, its boundary zonal velocity decreases to zero. One might intuitively expect to get $u=0$ for a stationary planet, ${ }^{2}$ although the temperature forcing still produces a zonally symmetric and circulation-generating global structure even without rotation: the planetary axis is no longer an axis of rotation, but the poles and the equator are still extrema of the forcing in this model. In the original theory, $\Omega=0$ is a singular point in parameter space; on either side of it, the maximum $|u|$ approaches the value given by (35), with the sign given by the sign of $\Omega$, and there is therefore a discontinuity in parameter space at $\Omega=0$. This is not a paradox in the strict sense, but one would expect the model to break down for sufficiently small $\Omega$ in reality (for example because the shear $\partial u / \partial \theta$

\footnotetext{
${ }^{2} \mathrm{We}$, in fact, find this to be the case in our numerical simulations described below. Although we do not show as many results for these $\Omega=0$ simulations, they are included as the hollow points on the vertical axis of Fig. 7a.
}

grows without limit as $\Omega \rightarrow 0$ ), whereas the variant theory does not have this particular problem.

\section{Numerical modeling}

\section{a. Simulation setup}

We performed simulations using Isca (Vallis et al. 2018), a code based on the Flexible Modeling System from the Geophysical Fluid Dynamics Laboratory (GFDL; Princeton). We integrate the hydrostatic primitive equations using a spectral dynamical core, at horizontal resolutions ranging from T42 to T341. ${ }^{3}$ The parameter values used (for atmospheric mass, gas constants, etc.) are those of Earth, except for rotation rate $\Omega$ where noted.

Thermal forcing and Rayleigh damping terms are as specified by Held and Suarez (1994), with additional horizontal $\nabla^{8}$ hyperdiffusion (for both temperature and velocity), whose coefficient (the hyperdiffusivity) is chosen to give a damping time of 0.1 day at the horizontal grid scale. This hyperdiffusion is intended to regularize the simulations while approximating the inviscid limit as closely as possible given the available resolution. Reducing the coefficient below this value can sometimes lead to model crashes. We have also verified in one case that doubling the horizontal resolution while keeping the physical hyperdiffusivity (instead of the gridscale damping time) fixed produces only a small change in results, and no further change when it is doubled again; this gives us adequate confidence that in our results which follow, with fixed gridscale damping time, when we obtain convergence as resolution is increased, we have converged to the inviscid limit.

Our simulations all use 30 vertical levels, unevenly spaced in $\sigma \equiv p / p_{s}$, where $p$ is the pressure and $p_{s}$ the surface pressure. No vertical damping is added. We have performed spot checks with different numbers of levels, and found that the only noticeable effect is on stratospheric wave activity (oscillations with vertical wavenumber at the grid scale), not the main tropospheric circulation with which we are concerned here (traces of such activity may be seen in the high-altitude streamfunction contours of Fig. 5k).

Several variants of the HH forcing have been used in idealized studies. In particular, static stability against small-scale convection may be introduced via a

\footnotetext{
${ }^{3}$ For our zonally symmetric simulations, the T for "triangular" is strictly inappropriate, because only the $m=0$ modes are present. However, rather than define a new and unfamiliar notation for our different spectral grids, we prefer to specify the corresponding base horizontal resolution first, and then state any further truncation to a reduced range of zonal modes.
} 
(a) $\Omega_{E}$

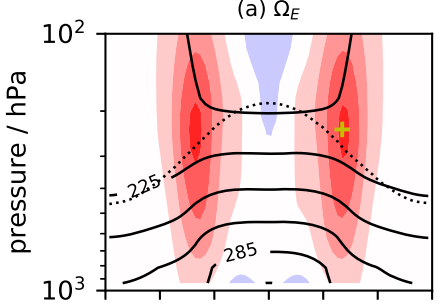

(e) $\Omega_{E}$ ( $m \leq 2$ only)

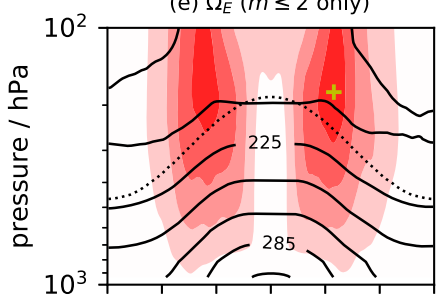

(i) $\Omega_{E}$ (m=0 only)

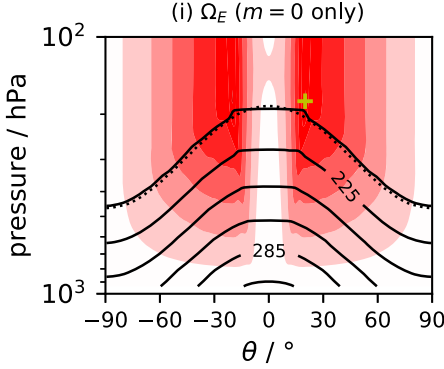

(b) $\Omega_{E} / 5$

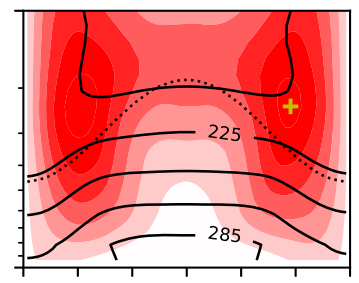

(f) $\Omega_{E} / 5$ ( $m \leq 2$ only)

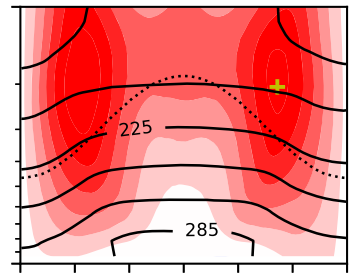

(j) $\Omega_{E} / 5$ ( $m=0$ only)

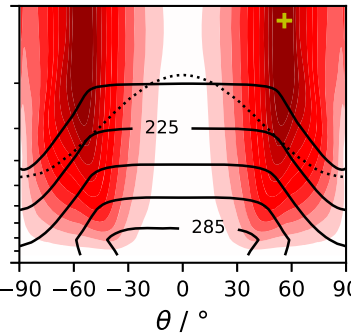

(c) $\Omega_{E} / 20$

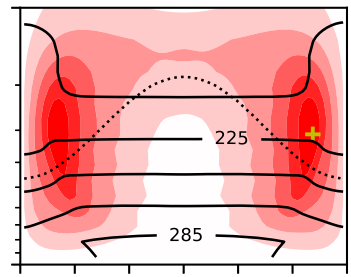

(g) $\Omega_{E} / 20$ ( $m \leq 2$ only)

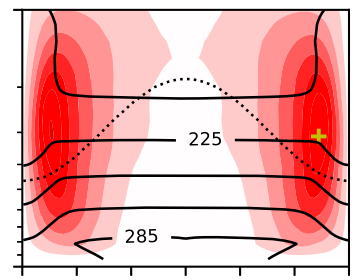

(k) $\Omega_{E} / 20$ (m=0 only)

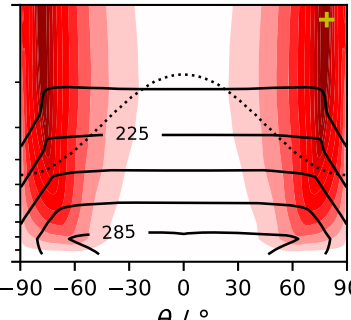

(d) $\Omega_{E} / 100$

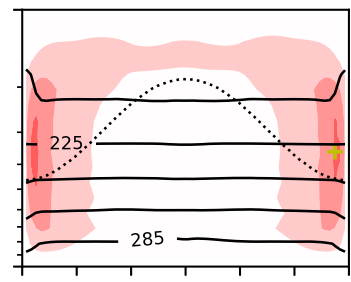

(h) $\Omega_{E} / 100$ ( $m \leq 2$ only)

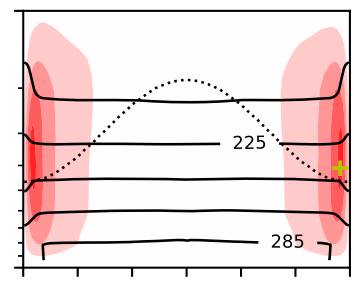

(I) $\Omega_{E} / 100$ ( $m=0$ only)

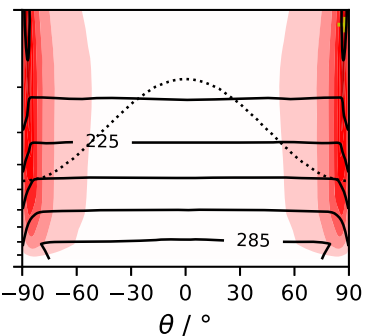

$\begin{array}{lllll}-75-50-25 & 0 & 25 & 50 & 75\end{array}$

$u / \mathrm{m} \mathrm{s}^{-1}$

FIG. 4. Zonal means against latitude $\theta$ and model-level pressure $\sigma p_{\text {ref }}(\mathrm{hPa})$ (only the bottom decade is shown), where $p_{\text {ref }}=10^{3} \mathrm{hPa}$ : zonal wind $u$ ( $\mathrm{m} \mathrm{s}^{-1}$; shading); temperature $T$ (solid contour lines; at $205 \mathrm{~K}$ and above in $20-\mathrm{K}$ intervals); forcing temperature $T_{\mathrm{eq}}$ (dotted contour line; at $205 \mathrm{~K}$ only); and the point of maximum $u$ within the Northern Hemisphere (NH) and the plotted pressure range (yellow plus sign). (a)-(d) Full 3D simulations, labeled by planetary rotation rate $\Omega$; (e)-(h) reduced simulations truncated to zonal wavenumbers $m=\{0,1,2\}$ only, for the same $\Omega$ values; and (i)-(1) zonally symmetric simulations truncated to zonal wavenumber $m=0$ only, for the same $\Omega$ values. $\Omega_{E}=7.3 \times 10^{-5} \mathrm{~s}^{-1}$ is the rotation rate of Earth. For each case, the highest available resolution (cf. Fig. 7) is used: (a)-(d) T42, (e)-(i) T170, and (j)-(l) T341.

latitude-dependent term, as by Held and Suarez (1994), or via a modified adiabatic index in the exponent relating temperature to potential temperature, as by Mitchell and Vallis (2010). Such functional forms would replace the $z$-dependent term in (2) of HH: but although this does determine the magnitude of the overturning circulation, it has played no role in our scaling analysis for the boundary latitude and zonal wind; it disappeared when vertically averaged to obtain our (12). In principle, more general forms would modify the above theory, but in practice the effect is small. In the simulations that we report here, we treat Held-Suarez forcing as effectively the same as HH. For more details, please see the appendix.

\section{b. Results}

Figure 4 shows simulations at four different rotation rates (left to right columns): Earth's $\Omega_{E}, \Omega_{E} / 5, \Omega_{E} / 20$, and $\Omega_{E} / 100$. Each panel shows the zonal wind (as color shading) and the temperature (as solid contour lines). Figure 4a is the Earthlike case. The rows correspond to fully 3D simulations (top row), reduced simulations truncated to zonal wavenumbers $m=\{0,1,2\}$ only (middle row, denoted W2), and zonally symmetric (ZS) simulations truncated to zonal wavenumber $m=0$ only (bottom row). These last are the simulations closest to the Held-Hou model.

The physical picture in terms of angular momentum, underlying the theory discussed above, is made more manifest in Fig. 5. This shows the same zonal-wind data as Fig. 4, but $u$ has been converted into the total zonal specific angular momentum $M=(u+\Omega a \cos \theta) a \cos \theta$, and plotted as $M / \Omega a^{2}-1$, where $\Omega a^{2}$ is the planetary specific angular momentum at the equator. This figure also shows contours of the overturning mass streamfunction. 
(a) $\Omega_{E}$

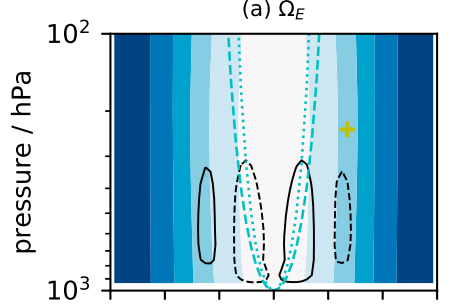

(e) $\Omega_{E}$ (m $m 2$ only)
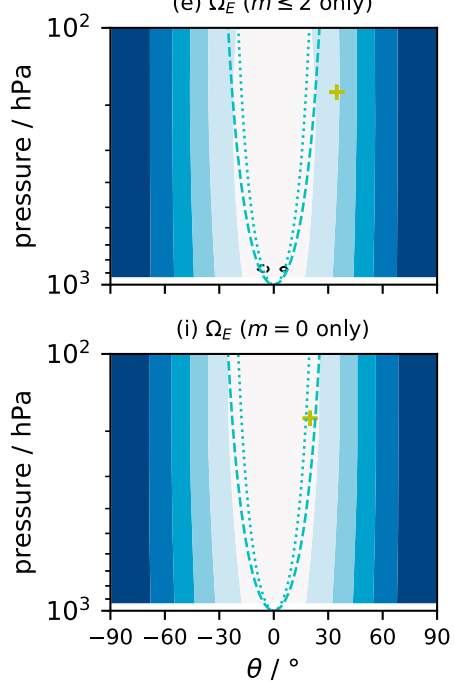

(b) $\Omega_{E} / 5$

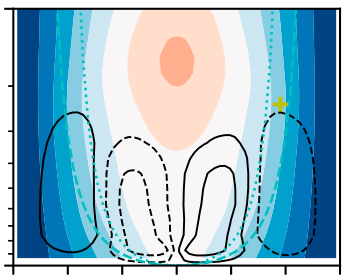

(f) $\Omega_{E} / 5$ ( $m \leq 2$ only)

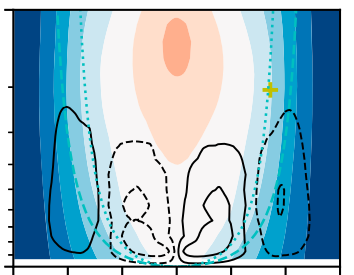

(j) $\Omega_{E} / 5$ ( $m=0$ only)

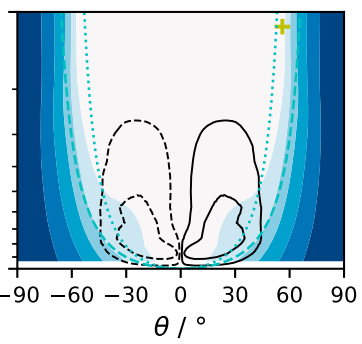

(c) $\Omega_{E} / 20$

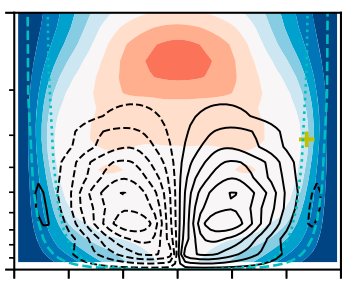

(g) $\Omega_{E} / 20(m \leq 2$ only)

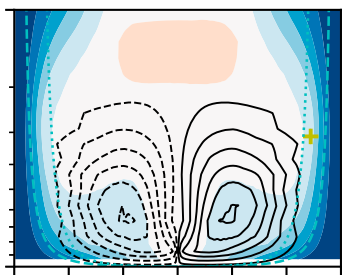

(k) $\Omega_{E} / 20(m=0$ only)

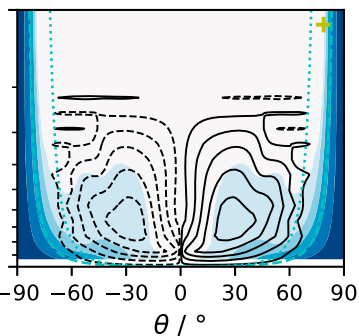

(d) $\Omega_{E} / 100$

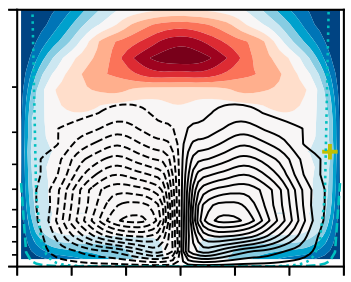

(h) $\Omega_{E} / 100$ ( $m \leq 2$ only)

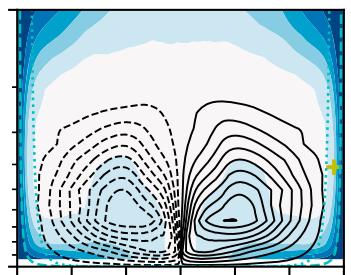

(I) $\Omega_{E} / 100(m=0$ only)

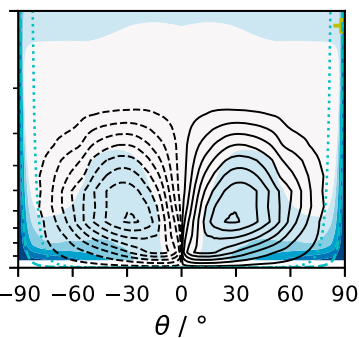

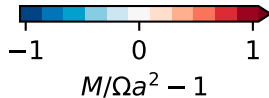

FIG. 5. As in Fig. 4, but with $u$ converted to the total zonal specific angular momentum $M=(u+\Omega a \cos \theta) a \cos \theta$ normalized to the equatorial planetary specific angular momentum $\Omega a^{2}$, where $a=6.4 \times 10^{6} \mathrm{~m}$ is the radius of Earth. Also shown is the mass streamfunction of the overturning circulation $\psi=2 \pi a \cos \theta \int \bar{v} d p / g$ (contour lines; interval: $1 \times 10^{11} \mathrm{~kg} \mathrm{~s}^{-1}$; equivalently, 22 levels between $\pm 1.05 \times 10^{12} \mathrm{~kg} \mathrm{~s}^{-1}$ inclusive; negative values are dashed). The cyan curves show the predicted Hadley cell height as a function of boundary latitude, as explained in the appendix — dashed and dotted lines correspond respectively to the theory with HH matching condition and the variant with continuous $u$.

Several trends are evident in these two figures. First, consistent with the above theory, the Hadley cell circulation pattern widens (and strengthens) as the planetary rotation reduces, eventually spanning almost the entire planet; the temperature flattens, and (consistent with thermal wind balance) the midlatitude zonal jets also move poleward. These observations are true not only for the zonally symmetric simulations but for all three rows. The white region in each panel of Fig. 5 is the region within which the zonal angular momentum is close to its equatorial surface value, that is, has been approximately conserved by the circulation. The red regions show superrotation. Notwithstanding the differences between the panels in this second figure, it is striking how simple the zonal wind appears when shown as $M$, compared with the complex structure of $u$ in the first figure. The additional structure in $u$ is purely geometric, as the expression for $M(u)$ makes clear.
Second, as the planetary rotation rate decreases, the zonal wind at first increases and then decreases, not only in the midlatitude jets but also at low latitudes for the 3D simulations, transitioning from retrograde equatorial winds in Fig. 5a to prograde winds in Fig. 5d, which features a wide superrotating layer. Figure $5 \mathrm{c}$ is similar to Titan, in which context such behavior has been studied by Mitchell and Vallis (2010) and later workers, who found that the superrotation is driven by nonlinear interactions between eddies (i.e., modes with nonzero zonal wavenumber $m$ ). Consistent with this, superrotation is absent in the corresponding zonally symmetric simulations. However, below the superrotating layer, the similarity between the $3 \mathrm{D}$ and the zonally symmetric simulations appears to become closer as the rotation rate reduces.

The simulations that include zonal modes $m=\{0,1,2\}$ only, shown on the middle row of each figure, are even 
(a) $\Omega_{E}$
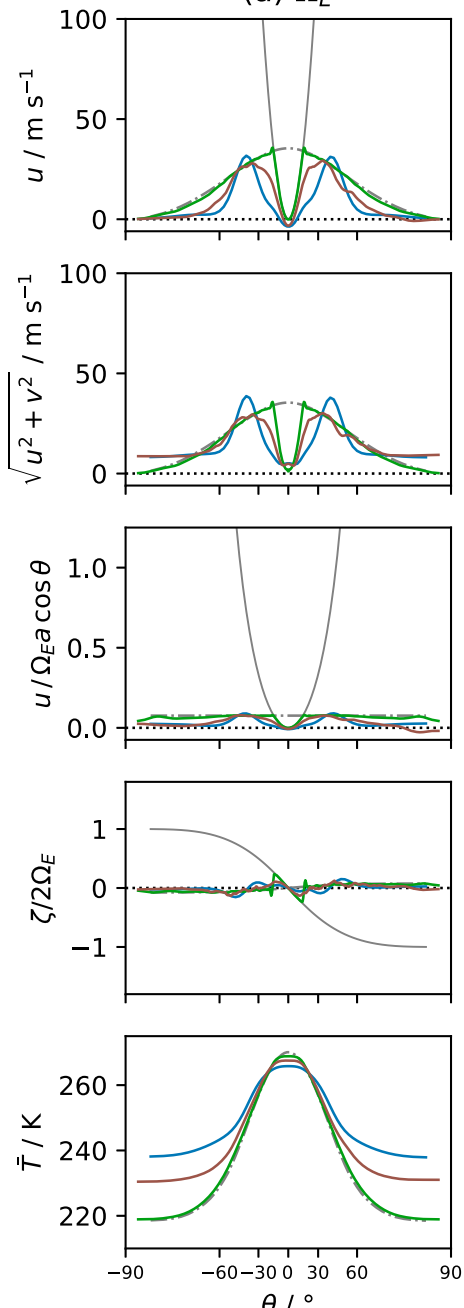

(b) $\Omega_{E} / 5$
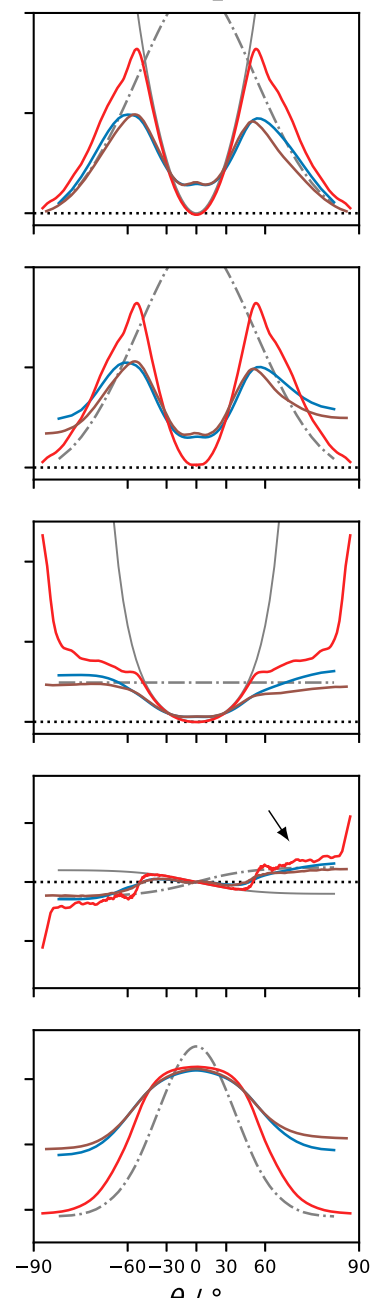

(c) $\Omega_{E} / 20$
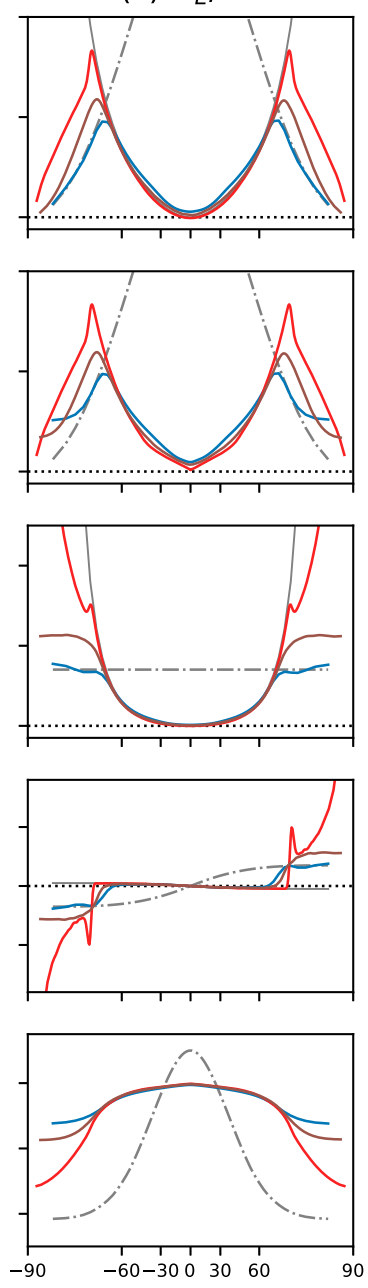

$\theta /^{\circ}$ (d) $\Omega_{E} / 100$
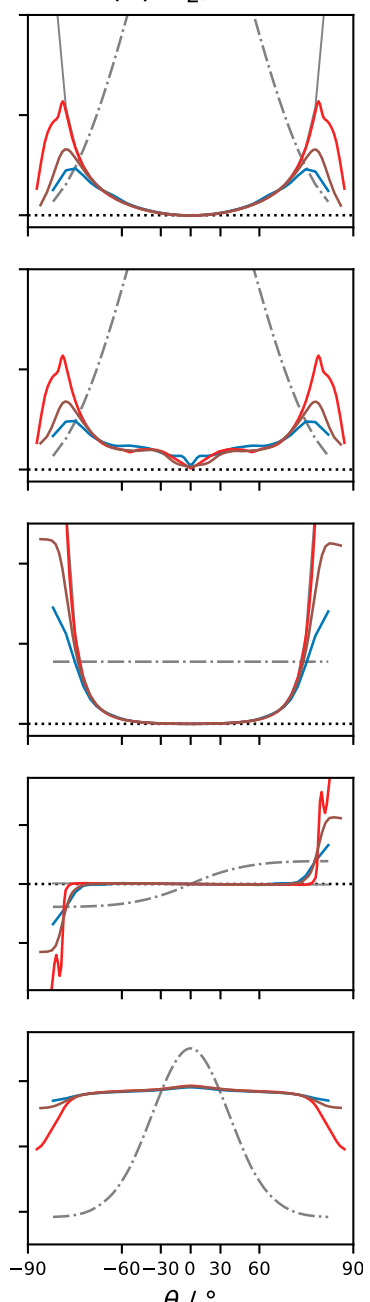

$\theta /^{\circ}$

FIG. 6. Horizontal profiles of zonal means of (top to bottom) zonal velocity $u$, horizontal wind speed, zonal velocity expressed as an angular velocity around the planetary axis, relative vorticity $\zeta$, and vertically averaged temperature $\bar{T}$, respectively, at model pressure level $\sigma p_{\text {ref }}=311 \mathrm{hPa}$. Columns are labeled by planetary rotation rate $\Omega$. Colors match the corresponding datasets shown in Fig. $7:$ blue for 3D (T42), brown for W2 (T170), and green or red for ZS (T170 or T341, respectively). Thin gray curves indicate the angularmomentum-conserving solution (solid) and the solution corresponding to thermal wind balance with the forcing temperature (dash-dotted), as explained in the appendix. To expand the polar regions smoothly, the horizontal axis is nonlinear in $\theta$ but linear in $\sqrt{90^{\circ}-|\theta|}$ (in each hemisphere).

closer to the full 3D simulations, and in particular are able to produce superrotating flow (although weaker than the corresponding $3 \mathrm{D}$ cases, at the lowest rotation rates). We use such reduced simulations to access higher resolutions than would be computationally feasible for fully 3D simulations, as discussed further in section $6 \mathrm{c}$. The most obvious discrepancy between the theoretical model and these results - and again this applies to all three rows-is that the temperature does not reduce sharply to the forcing temperature at the edge of the Hadley cell. In each panel of Fig. 4, the lowest temperature contour shown is the $205-\mathrm{K}$ contour, and the $205-\mathrm{K}$ forcing contour is shown dotted. They only overlay at all in Fig. 4i, in which they overlay everywhere because the overturning circulation is so weak.

It is also clear in the simulation results that the zonal wind does not suddenly jump to a smaller value at the Hadley cell edge. Figure 6 shows horizontal profiles of the zonal wind $u$ (top row) at a single selected model level near the top of the overturning circulation. The zonal velocity $u$ does track $u_{M}$ (marked by the solid gray line) well in the Hadley cell region, especially in the zonally symmetric cases (green and red curves) and at the lowest rotation rate (rightmost column). However, it does not discontinuously jump to the equilibrium 
thermal wind (marked by the dash-dotted gray line) in the poleward region. There is a sharper change in its gradient $\partial u / \partial \theta$, but the gradient on either side of the maximum is of similar magnitude (unlike in Fig. 1 at the dashed vertical lines); see the final paragraph of section 2 and of section 3. So it appears that both the zonal wind varies more smoothly than in the original theory, and the temperature varies more smoothly than in either theory.

The new matching condition may appear to be even more unphysical than the first, because a discontinuity in $\bar{\Theta}$ is problematic for thermal wind balance, even with equal gradients $\bar{\Theta}^{\prime}$ on either side. We might attempt to fix this in the theoretical model by adding a constant to $\bar{\Theta}$ in the polar region to close the discontinuity, which does not change the zonal wind (since thermal wind balance involves only the gradient), but such a fix does mean that $\bar{\Theta}=\bar{\Theta}_{E}$ no longer holds there. Hence, from (5), the polar region is no longer circulation free in steady state. The closure condition, (20), will now include a contribution from the polar region; however, as mentioned at the end of section 5 , in the low- $\Omega$ limit its contribution will be negligible, both because the boundary latitude is close to $\theta=\pi / 2$ and because the weighting factor $\cos \theta$ is small. In other words, a tiny adjustment to the Hadley cell covering the rest of the planet would bring the system back into global energy balance.

More generally, any adjustment to the temperature would make a negligible contribution to (20) in the low- $\Omega$ limit. The adjustment would not, however, reproduce the same $u_{E}$, and hence would not in general leave the boundary latitude or the polar vorticity unaffected. Since a generalized model of this form is underdetermined in the absence of any additional principles, a more comprehensive understanding of the polar region is needed. Before addressing this issue further in section $6 \mathrm{~d}$, we compare the simulation results with the theoretical low- $\Omega$ scalings more quantitatively.

\section{c. Testing the theoretical scalings}

We now compare the theoretical scalings for the $\mathrm{HH}$ theory and the continuous- $u$ variant with our numerical results; the comparison is shown in Fig. 7. The data points plotted include many more runs than those shown in Figs. 4 and 5. Figure 7 includes intermediate and lower rotation rates, and also different horizontal resolutions (indicated by different marker sizes) for the reduced (W2: $m \leq 2$ only) and ZS ( $m=0$ only) datasets. It can be seen (where the green and red discs coincide) that the zonally symmetric runs are converged in horizontal resolution, which corresponds to the inviscid limit, down to approximately $\Omega=\Omega_{E} / 100$. We discuss convergence further in section 6e below.

Our simulations use a hydrostatic primitive model rather than a Boussinesq model. Caballero et al. (2008) developed the Held-Hou theory for a compressible atmosphere with a more sophisticated radiative model, and found similar scaling behavior (but did not focus on low rotation rates). Here we take $\Delta_{H} g H \rightarrow \Delta T_{y} R_{d}$, where $\Delta T_{y}=60 \mathrm{~K}$ is the Held-Suarez equator-pole temperature difference at the surface ${ }^{4}$ and $R_{d}=$ $287 \mathrm{~J} \mathrm{~kg}^{-1} \mathrm{~K}^{-1}$ is the gas constant for dry air. (Since the panels of Fig. 7 are $\log -\log$ plots, this value sets the intercepts only; it does not affect the slopes.)

The Held-Suarez forcing also features a stratospheric cap temperature of $200 \mathrm{~K}$, below which the forcing does not drop at higher altitudes. We have not needed to take additional account of this; it effectively sets the tropopause height at about the pressure scale height (for Earth). A general theory for other planets will need to pay more attention to this distinction; on Venus for example there are several scale heights between the tropopause and the surface.

Figures 4 and 5 mark with a plus sign in each panel the Northern Hemisphere maximum of $u$. In the zonally symmetric theories discussed above, the maximum $u$ occurs at the boundary between the low- and highlatitude regions, and so we take this maximum to define the boundary (co)latitude in our simulations.

We tested these theories by performing a scan in $\Omega$ and plotting the boundary colatitude $\varphi_{H}$; the maximum zonal wind $u_{H}$; and the maximum zonal wind expressed as an angular velocity around the planetary axis, $\omega_{H} \equiv u_{H} / a \sin \varphi_{H}$. These are shown in Figs. $7 \mathrm{~b}-\mathrm{d}$, along with the predicted low- $\Omega$ scalings from section 5 , also shown as gray lines in the same panels of Fig. 3.

In general we see that the simulations fall between the predictions of the two sets of scalings. In Fig. 7c, the boundary colatitude follows the HH scaling quite well in the low-rotation limit for the ZS simulations, provided sufficient resolution is used (higher $\mathrm{T}$ number). The 3D simulations appear closer to the continuous- $u$ prediction, but this result is not conclusive because of their limited resolution. Figures $7 \mathrm{~b}$ and $7 \mathrm{~d}$ tend to corroborate this story to some degree; in all simulations the maximum zonal wind does eventually decrease as rotation rate decreases, but it is sustained at a higher level, which is especially clear when viewed as an angular velocity in Fig. 7d, for the zonally symmetric simulations at high resolution. We cannot be certain that the runs at the lowest nonzero rotation rate,

\footnotetext{
${ }^{4} \Delta T_{y}$ (in K) is called "delh" in the code.
} 
(a)

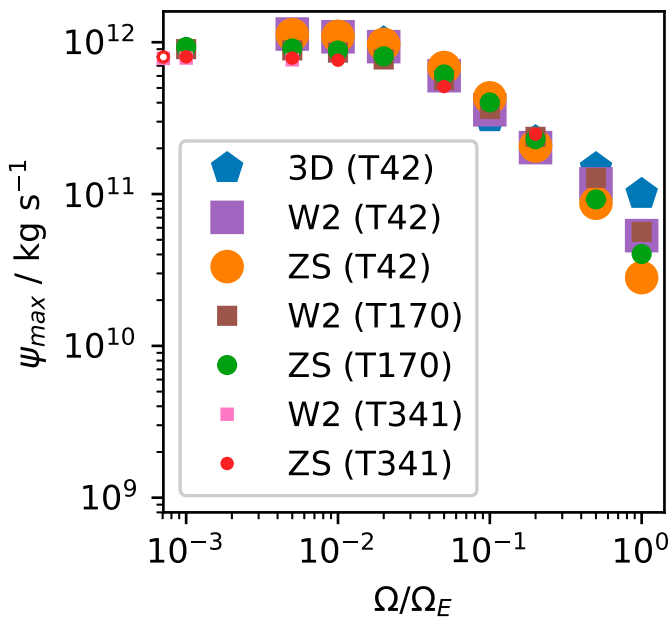

(c)

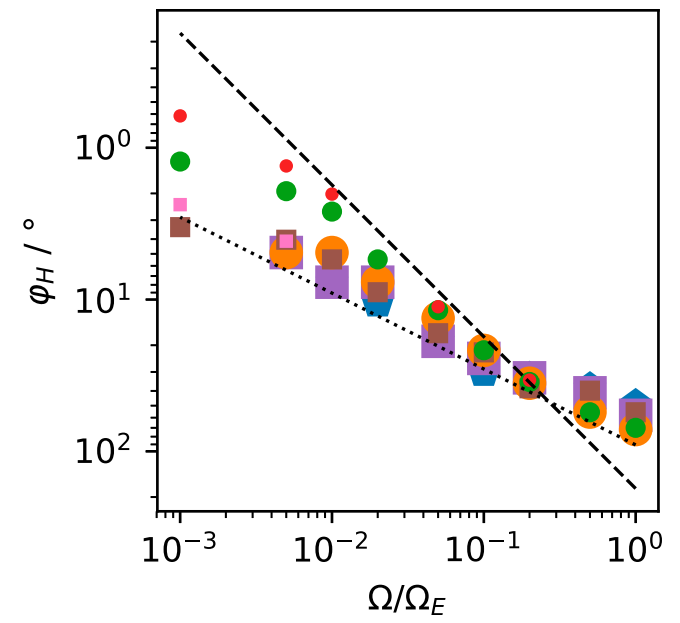

(b)

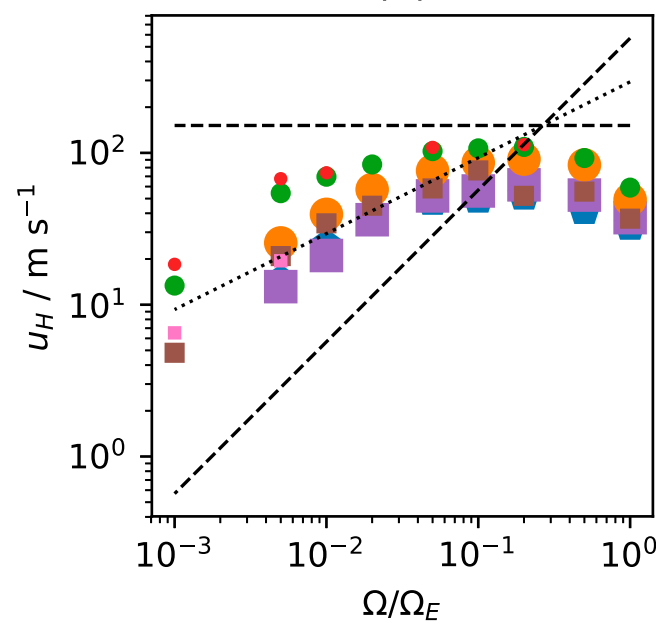

(d)

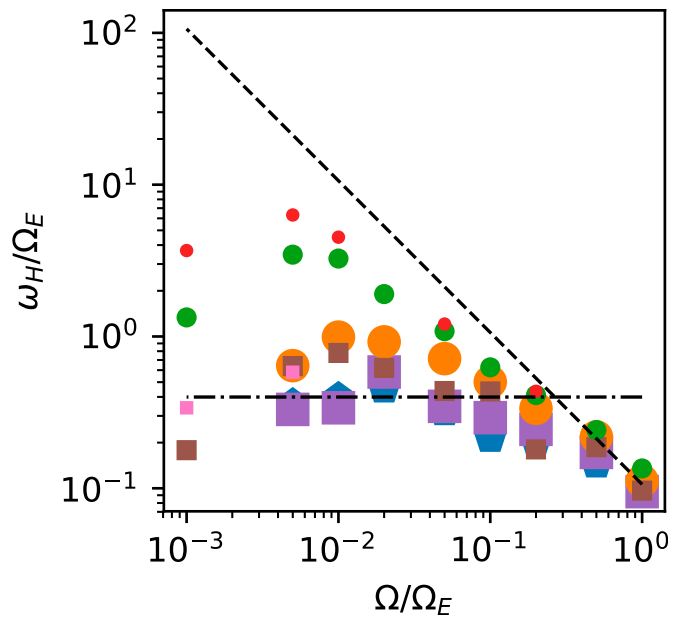

FIG. 7. (a) The magnitude of the hemispheric overturning circulation, as given by the maximum of the mass streamfunction $\psi$, vs planetary rotation rate $\Omega$. (b) Maximum (in latitude) of the zonal wind $u_{H}$. (c) Hadley cell boundary colatitude $\varphi_{H}$. (d) Maximum zonal wind as an angular velocity around the planetary axis $\omega_{H} \equiv u_{H} / a \sin \varphi_{H}$. The boundary colatitude is defined to be the colatitude of the point of maximum zonal wind, indicated by the plus signs in Figs. 4 and 5. Various datasets are plotted, as indicated in the legend of (a): fully 3D simulations, at T42 resolution (3D; pentagons); simulations truncated to zonal wavenumbers $m=\{0,1,2\}$ only (W2; squares), at various resolutions (differentiated by marker size); and zonally symmetric simulations truncated to zonal wavenumber $m=0$ only (ZS; discs), again at various resolutions. In (a) the limiting value from W2 and ZS simulations at exactly $\Omega=0$ is shown (hollow points on the vertical axis). The dashed lines show the low- $\Omega$ limit of the original HH theory. In (b) there are two such lines corresponding to the discontinuity in $u$ : the upper line is on the equatorward side and the lower line is on the poleward side. The dotted lines show the low- $\Omega$ limit of the modified theory with the alternative continuous- $u$ matching condition. In (d), the lower dashed line and the dotted line coincide and are shown as a dash-dotted line.

$\Omega=\Omega_{E} / 1000$, are converged in resolution even for the highest resolution shown here, so we include these points for completeness only. It is interesting that at the next lowest rotation rate, $\Omega=\Omega_{E} / 200$, the $\mathrm{W} 2$ runs do appear to be very well converged in resolution (pink square overlaying brown square), and much closer to the continuous- $u$ theory. It is a question for further work to determine if this is indeed representative of fully 3D (and thus much more expensive) runs that are converged in resolution.

\section{d. Further discussion}

Figure 7 a shows the magnitude of the overturning circulation in the Hadley cell $\psi_{\max }$, where $\psi$ is the mass 
(a) $\Omega_{E}$

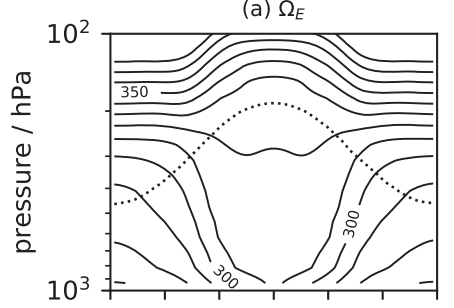

(e) $\Omega_{E}$ ( $m \leq 2$ only)
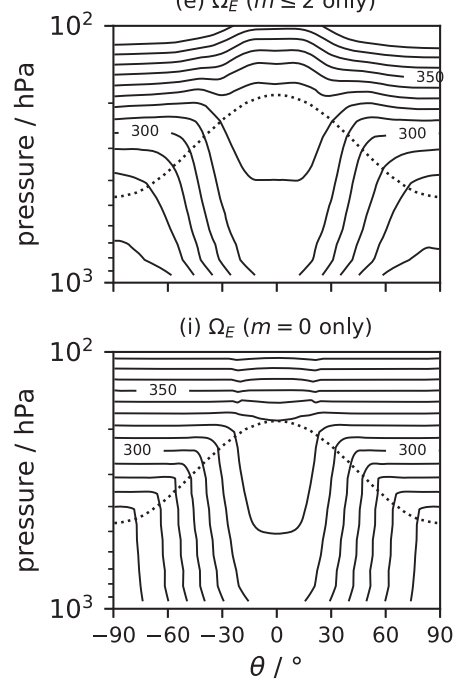

(b) $\Omega_{E} / 5$

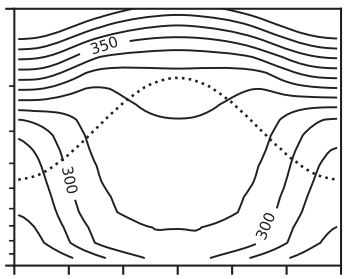

(f) $\Omega_{E} / 5$ ( $m \leq 2$ only)

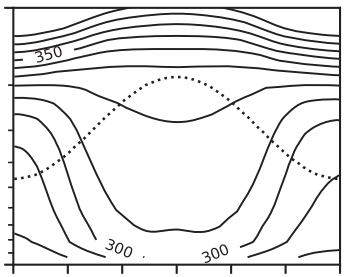

(j) $\Omega_{E} / 5$ ( $m=0$ only)

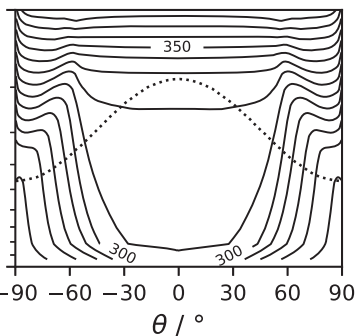

(c) $\Omega_{E} / 20$

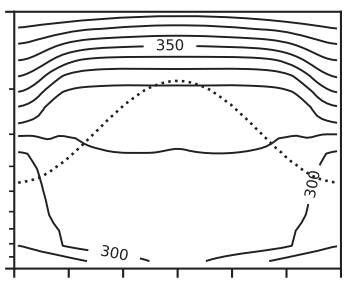

(g) $\Omega_{E} / 20(m \leq 2$ only)

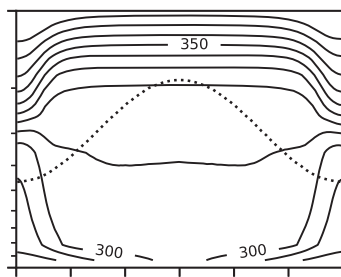

(k) $\Omega_{E} / 20$ ( $m=0$ only)

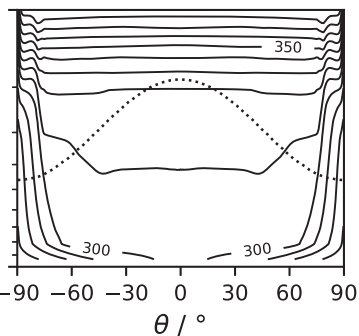

(d) $\Omega_{E} / 100$

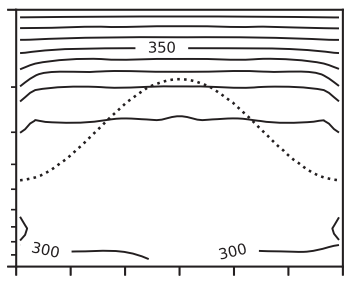

(h) $\Omega_{E} / 100$ ( $m \leq 2$ only)

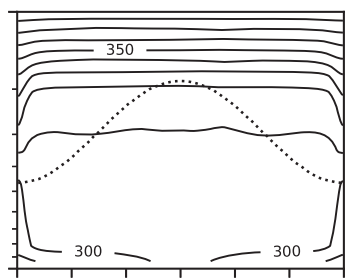

(I) $\Omega_{E} / 100(m=0$ only)

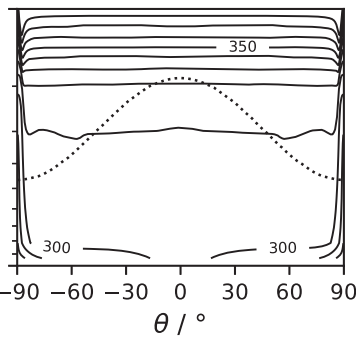

FIG. 8. As in Fig. 4, but for potential temperature $\Theta$ (solid contour lines, $260-380 \mathrm{~K}$ in $10 \mathrm{~K}$ intervals).

streamfunction. On this panel are also shown, as (two coincident) hollow points on the vertical axis, the results from W2 and ZS simulations at exactly $\Omega=0$. These simulations have $u=0$ everywhere, so they do not appear on the other three panels of Fig. 7. Figure 7a supports the point made earlier, that the overturning circulation is in good agreement between all three sets of runs (3D, W2, and $\mathrm{ZS}$ ), with this agreement improved at low rotation rates and converging to a nonzero value at $\Omega=0$.

Estimates of the overturning time may be obtained from $m_{H} / \psi_{\max }$, where $m_{H}$ is the mass of the Hadley cell, or $a \theta_{H} / v$, or $H / w$. In the low- $\Omega$ limit, these are all of similar order to the Held-Suarez damping time scale (which is 40 days in the bulk of the atmosphere). Thus, in this limit the circulation is not in the very weak regime considered by $\mathrm{HH}$; it is strong enough to drive the temperature toward the dry adiabatic lapse rate of constant potential temperature, as is demonstrated by Fig. 8 .

We noted above that more general modifications to the polar temperature may be possible within the same theoretical framework, but would change the form of $u_{E}$ and hence the polar vorticity. Figure 6 does suggest that the continuous- $u$ theory may have some validity: it is especially evident in Fig. 6b that $|\partial u / \partial \theta|$ is similar on either side of the maximum $u$. Figure $7 \mathrm{~d}$ clearly shows the boundary angular velocity to be sustained within an order of magnitude of $\Omega_{E}$, and therefore many orders of magnitude above low values of $\Omega$. However, while in some cases there is a distinct plateau in the angular velocity and the vorticity (third and fourth rows of Fig. 6) on the poleward side of the point of maximum $u$, for example, where indicated by the arrow, Fig. 6 exhibits only limited support for rigid-body rotation across the whole of the polar region, which is a feature common to both of the zonally symmetric theories considered here (and indicated by the gray dashdotted straight line in the middle row). One may surmise that in the zonally symmetric cases (green and red), the temperature that was raised on the poleward side to achieve continuity at the boundary is then required to return toward the equilibrium temperature at the pole, and is thus steepened, resulting in the even higher angular velocities and vorticities observed poleward. In the zonally symmetric case there can be no cross-pole flow. In the 3D and W2 cases, however, there can be, and so the temperature is not as constrained to steepen, and the poleward vorticity can remain close to the boundary value. The relative 
(a)

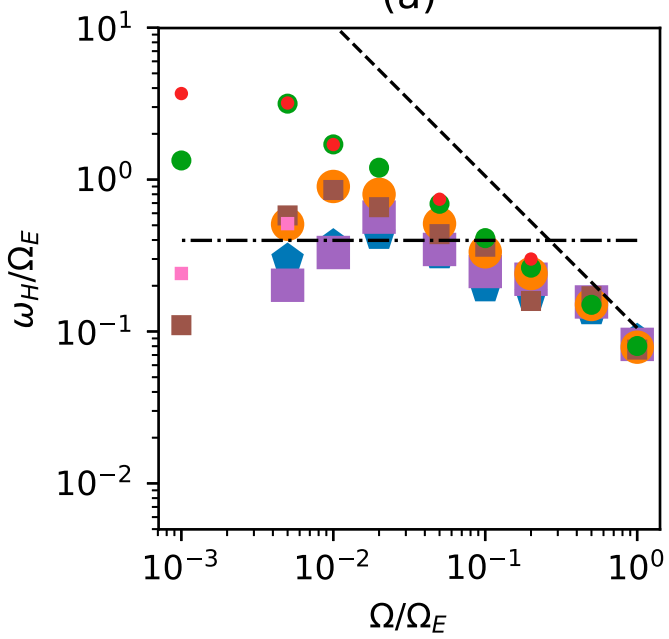

(b)

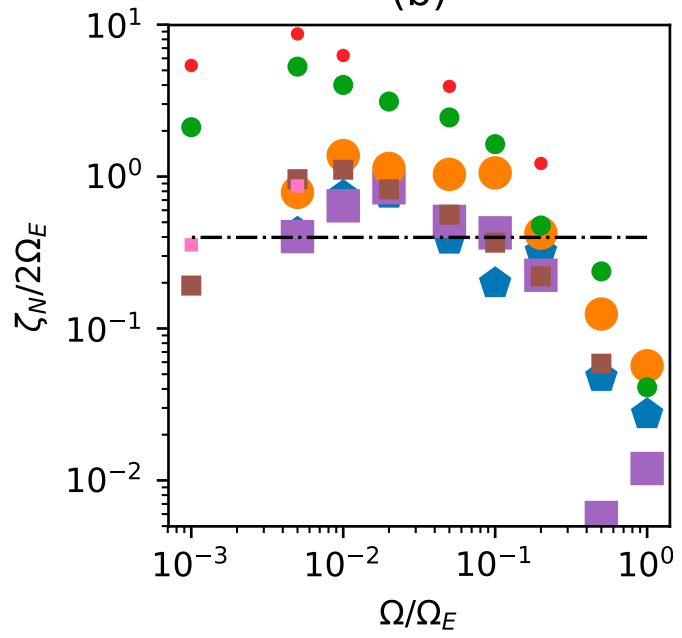

FIG. 9. As in Fig. 7, but considering only the model pressure level $\sigma p_{\text {ref }}=311 \mathrm{hPa}$. (a) The maximum zonal wind as an angular velocity around the planetary axis $\omega_{H}$. (b) The relative vorticity at the north pole $\zeta_{N}$, normalized to give an angular velocity in the same units of $\Omega_{E}$. The dashed line again represents the angular velocity in the low- $\Omega$ limit of the $\mathrm{HH}$ theory on the equatorward side of the Hadley cell boundary, which is not equal to the vorticity there in the $\mathrm{HH}$ theory $(\zeta=-f)$, and the pole is on the other side of the boundary, so there is no dashed line in (b).

steepening of the temperature profile in the ZS cases can be seen in the middle columns of Fig. 4, as well as in the bottom row of Fig. 6, in which the vertical average $\bar{T}=\int_{p_{s}} T d(\ln p) / \int_{p_{s}} d(\ln p)$ [cf. (A2) in the appendix] is plotted. The cross-pole flow in the 3D and $\mathrm{W} 2$ cases can be seen in the wind speed profiles in the second row of Fig. 6 (blue and brown curves), which do not go to zero at the poles while the $u$ profiles do. For all the runs, Fig. 9a shows the angular velocity at the point of maximum zonal wind taken over the single model level shown in Fig. 6, and Fig. 9b shows the vorticity evaluated at the north pole itself (in equivalent units). In the higherresolution ZS cases (green and red discs), the polar vorticity is elevated close to the level corresponding to rigid-body rotation at the equatorward boundary value from the $\mathrm{HH}$ theory (dashed line), whereas the other cases are closer to the predicted poleward value (dash-dotted line).

Neither zonally symmetric theory could apply exactly to a full GCM simulation, in which one would not expect actual discontinuities to be maintained. Since eddies may in general play a role in removing discontinuities (or opposing their creation), via turbulent diffusion for example, but such a mechanism is not possible in a zonally symmetric simulation, some other mechanism must operate in this case-and may or may not also be the dominant mechanism operating in $3 \mathrm{D}$ simulations. Here, we see that the ZS simulations do seem to be distinguished from the other two sets, but it is also interesting that the apparently underresolved ZS simulations at T42 are in better agreement with the $3 \mathrm{D}$ and W2 cases; so perhaps gridscale numerical dissipation at low resolutions is mimicking the role of small-scale eddies. Fang and Tung (1994) have discussed the relevance of viscous solutions that may represent a parameterization of eddies in this manner. (The actual molecular transport coefficients are much too small for molecular viscosity or heat conduction to play a role in the "general" circulation, that is to say on scales that are resolvable in any simulation of the whole planet.)

As a final note, we observe that the 3D cases exhibit both $u(0)<0$ and $u(0)>0$ on occasion (blue curves for $\Omega=\Omega_{E}$ and $\Omega=\Omega_{E} / 5$ respectively in Fig. 6).

\section{e. Circulation poleward of the zonal-wind maximum}

We do not have a full understanding of the polar overturning circulation, but it may be characterized to some extent by the consideration of additional diagnostics. We focus first on the $\Omega_{E} / 5$ case.

The zonal-mean zonal momentum equation for the numerical model may be written in the form

$$
\begin{aligned}
\frac{\partial \bar{u}}{\partial t}+\underbrace{\bar{v}\left(\frac{1}{a} \frac{\partial \bar{u}}{\partial \theta}-f-\frac{\bar{u} \tan \theta}{a}\right)}_{(\mathrm{b})}+\underbrace{\frac{1}{a} \overline{\left(\overline{v^{\prime} \frac{\partial u^{\prime}}{\partial \theta}}-\overline{v^{\prime} u^{\prime}} \tan \theta\right)}}_{(\mathrm{c})} \\
+\underbrace{\bar{\omega} \frac{\partial \bar{u}}{\partial p}+\overline{\omega^{\prime} \frac{\partial u^{\prime}}{\partial p}}}_{(\mathrm{d})}+\underbrace{k_{f} \bar{u}}_{(\mathrm{e})}+D=0,
\end{aligned}
$$

where the overbar and prime indicate mean and fluctuating quantities respectively $\left(u=\bar{u}+u^{\prime}\right.$ etc.), $\omega \equiv D p / D t$ 
(a) horizontal (total)

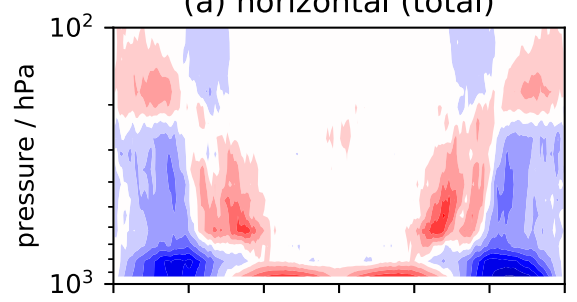

(d) vertical (total)

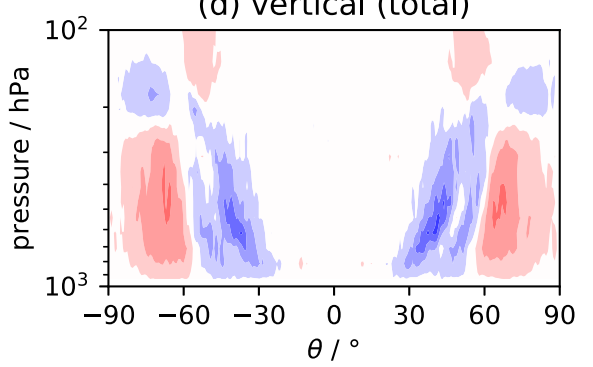

(b) horizontal (mean)

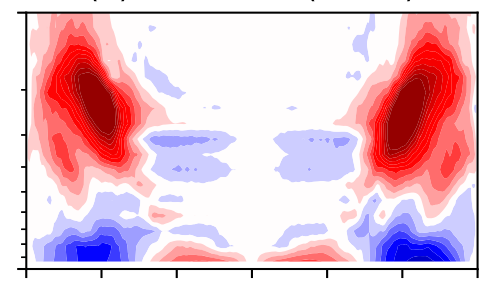

(e) Rayleigh damping

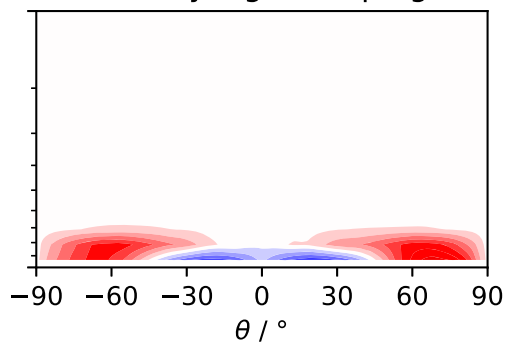

(c) horizontal (eddy)

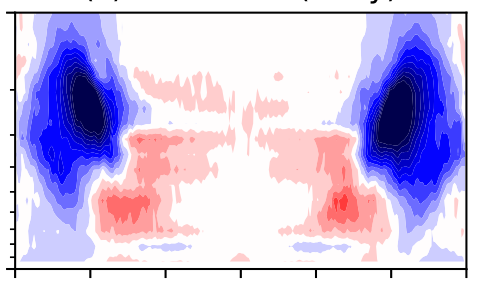

(f) residual

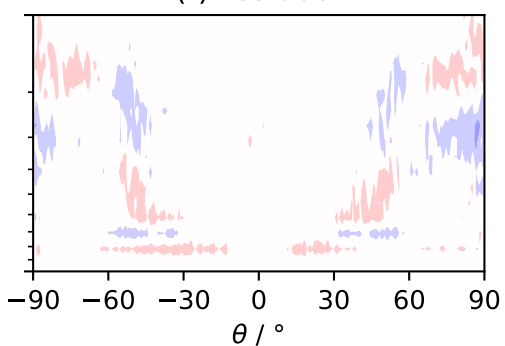

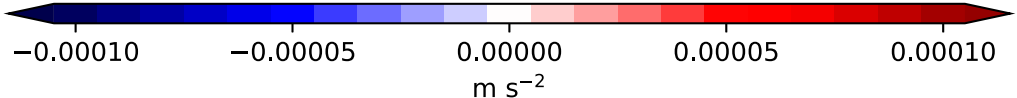

FIG. 10. Zonal- and time-mean contributions to the evolution of the zonal wind $u$ in the $\Omega_{E} / 5$ case W2 (T170) against latitude $\theta$ and model-level pressure $(\mathrm{hPa})$. See section $6 \mathrm{e}$ for an explanation of the various panels.

is the vertical pressure velocity, $k_{f}$ is the Rayleigh damping coefficient, and $D$ represents the spectral damping (hyperviscosity). The bracketed terms, when time averaged, correspond to panels of Fig. 10 (W2) and Fig. 11 (ZS): (b) includes the Coriolis term $-f \bar{v}$ and the mean-flow contribution to the meridional advection and metric terms; (c) is the corresponding fluctuation contributions, and (a) $\equiv(b)+(c)$. These are all labeled "horizontal" terms. The vertical advection is taken together as (d), that is, $\overline{\omega \partial u / \partial p}$; (e) is the Rayleigh friction; and the residual (f) accounts for spectral damping and any numerical errors.

The dichotomy (6) is equivalent to (a) $=(\mathrm{b})=(\mathrm{c})=0$, since there are neither spatial nor temporal fluctuations in a zonally symmetric steady state. Looking first at Fig. 11, our zonally symmetric case, we see that this dichotomy holds well in the Hadley cell region (center), but is violated in the region poleward of the Hadley cell. Of course there are no eddies in a zonally symmetric model, but here we define the mean indicated by overbar to be a time mean as well as a zonal mean; then the fluctuating terms consist of the time-varying contributions, and we can see that the total (a) is dominated by these fluctuations (c). The nonvanishing horizontal terms are balanced by the vertical advection (d), and by Rayleigh friction in the surface layer.

The dichotomy is an exact result in steady state for the zonally symmetric shallow-water model of Adam and Paldor (2009), who drew attention to the existence of an unsteady layer between the two regions satisfying (6), and to the fact that while in principle this layer narrows with time, the process may be too slow to follow (or presumably to matter relative to other processes, such as the seasonal time scale, in a real-world context; cf. Fang and Tung 1999). Here, we may be seeing a related phenomenon in three dimensions. We find no evidence that the fluctuation level decreases, even slowly, in the statistically steady state. The fluctuations span a significant fraction of the latitude range, and this is consistent with the deviation of the profiles in Fig. 6 from the equilibrium solution across the whole poleward region. Figure 12 shows the fluctuation range in time at a single model pressure level. It can also be seen in this figure that, while increasing the horizontal resolution allows the peak in the time-averaged $u$ to be resolved more sharply, ${ }^{5}$ the deviation in the poleward region from the circulation-free equilibrium (which is indicated by the dash-dotted gray curve) appears to be converged.

\footnotetext{
${ }^{5}$ Low resolution may artificially widen a narrow "inner viscous" boundary layer discussed by Fang and Tung (1996). Adam and Paldor (2009) point out that the nature of any jump depends on the details of the numerical scheme, and our spectral scheme will prevent any exact discontinuity, even in the gradient. However, regardless of the numerical scheme and the spatial resolution, it is unsurprising that the discontinuity of the steady-state theory is smoothed out in the time average of the fluctuations.
} 


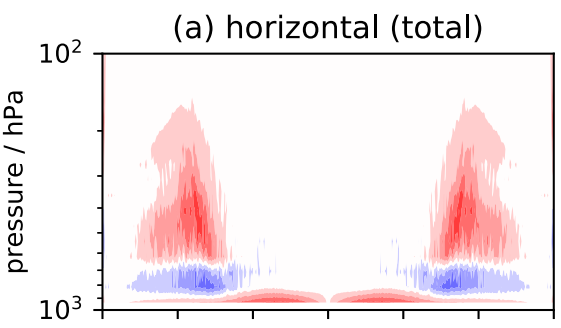

(d) vertical (total)

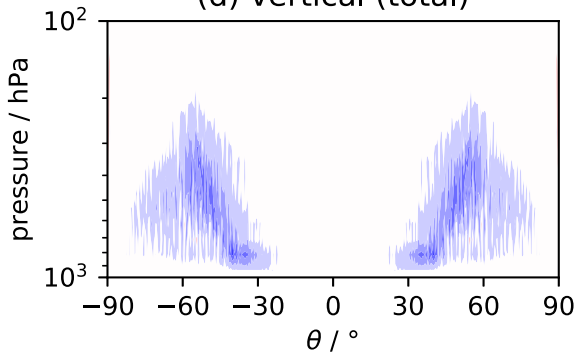

(b) horizontal (mean)

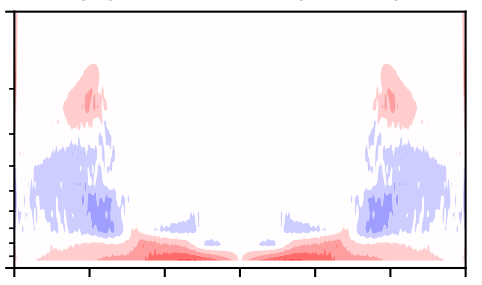

(e) Rayleigh damping

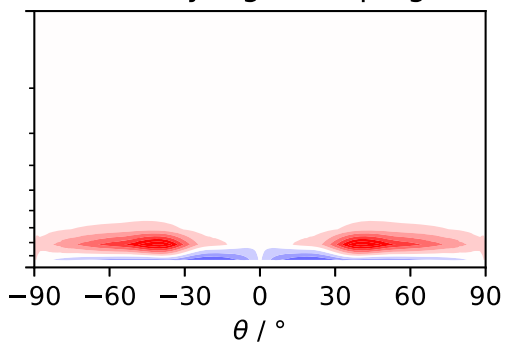

(c) horizontal (fluctuating)

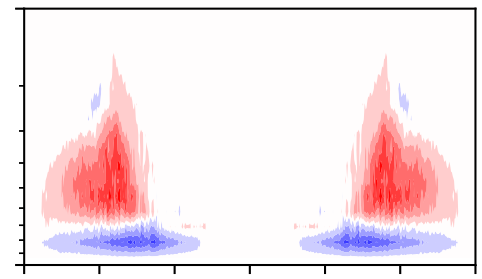

(f) residual

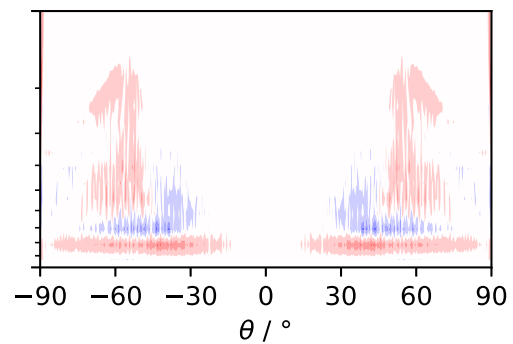

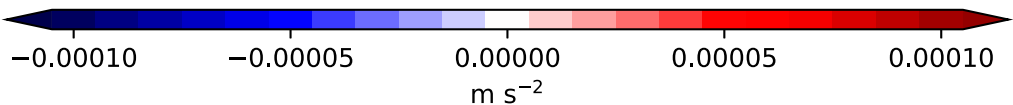

FIG. 11. As in Fig. 10, but for ZS (T341). In this case there are no spatial eddies (the zonal mean captures everything), but (a) the horizontal total is partitioned into the contributions from (b) the time mean and from (c) the fluctuations (in time).

In the W2 case, Fig. 10, again the overall balance is between the horizontal and vertical terms, and by Rayleigh friction in the surface layer; again the dichotomy holds well in the Hadley cell region when the total (a) is considered. In this case, however, the fluctuations are due essentially in their entirety to spatial eddies (so that defining the overbar to be just the zonal mean makes no visible difference to the figure), and there is a large cancellation between the mean flow (b) and the eddies (c), in the region where the reversed overturning circulation is visible in the streamfunction contours of Fig. 5f. Some cancellation also occurs in the Hadley cell region.

The violation of (6) in the horizontal terms and the balancing vertical advection implies an overturning circulation, ${ }^{6}$ which is not obvious outside the Hadley cell region in the zonally symmetric case, Fig. $5 \mathrm{j}$. We therefore include an additional figure showing the streamfunction in much more detail, and highlighting the low-value contours: this is Fig. 13b. The extension of the direct cell into the poleward region at low altitude is clear, as is a weak reversed circulation aloft. For comparison, in Fig. 13a, we show the transformed Eulerian

\footnotetext{
${ }^{6}$ Vertical motion is necessarily associated with nonzero horizontal divergence, by mass conservation. The divergent part of the meridional wind can be unambiguously attributed to a meridional overturning circulation: compare with (4) of Zhang and Wang (2013).
}

mean (TEM) streamfunction for the W2 case. (Both panels in fact show the TEM streamfunction, since the TEM and the usual Eulerian mean are identical for the ZS case.)

The meridional overturning mass streamfunction used for the numerical results in this paper is

$$
\psi=\frac{2 \pi a \cos \theta}{g} \int \bar{v} d p
$$

The corresponding TEM mass streamfunction is given by ${ }^{7}$

$$
\psi^{*}=\psi-\frac{2 \pi a \cos \theta}{g} \frac{\overline{v^{\prime} \Theta^{\prime}}}{\partial \bar{\Theta} / \partial p} .
$$

Here the overbar indicates the zonal mean, but in Fig. 13a the time mean has also been taken, in order to smooth out noise in the denominator.

In the $\mathrm{W} 2$ case, the reversed mean-flow overturning circulation is clear in Fig. 5f, but the TEM framework

\footnotetext{
${ }^{7}$ The TEM formulation for the primitive equations on a sphere is taken from appendix A of chapter 15 of Vallis (2017). Here we have converted from log-pressure to pressure coordinates. In both (40) and (41) we incorporate the geometric prefactor appropriate for the mass streamfunction: $2 \pi a \cos \theta$ is the circumference of the latitude circle across which $v$ flows. The meridional mass flux circulating between two zonally symmetric toroidal $\psi$ surfaces is then simply the difference between the two $\psi$ values.
} 


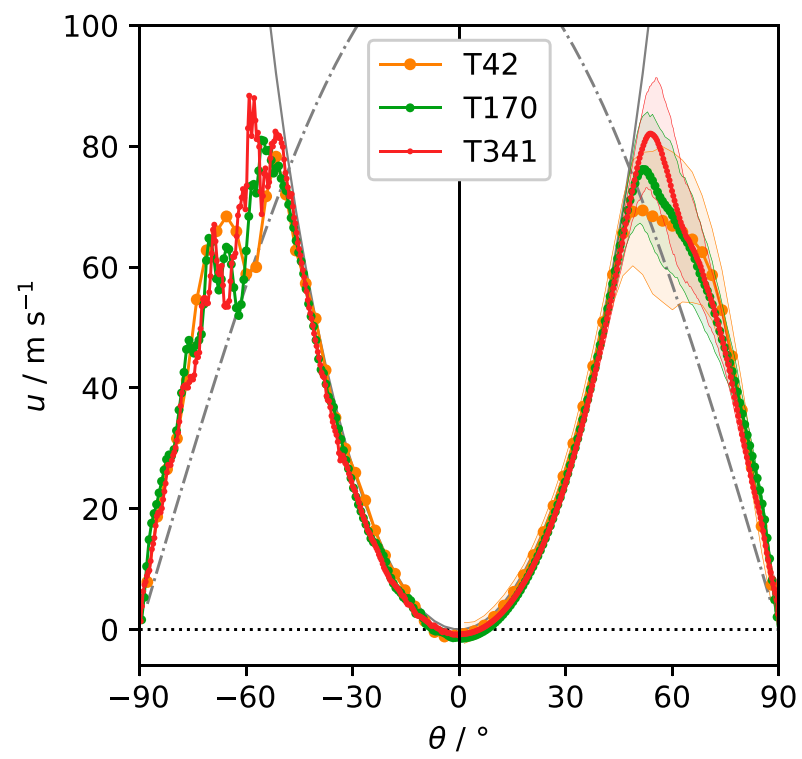

FIG.12. Horizontal profiles of the time-mean zonal velocity $u$ at model pressure level $\sigma p_{\text {ref }}=311 \mathrm{hPa}$ for $\Omega=\Omega_{E} / 5$, averaged (left) over the final day in the Southern Hemisphere and (right) over 1050 days in the Northern Hemisphere, for ZS runs at the indicated resolutions. The gray curve and thick red curve (right panel) are as in the top panel of Fig. 6b, although a linear latitude scale is used here. The spatial grid points are marked on each thick curve to give an indication of horizontal resolution, although it should be noted that the number of points exceeds the maximum wavenumber (usually denoted $\ell$, as in $Y_{\ell}^{m}$ for the spherical harmonic) by about $50 \%$ (e.g., there are 64 points at T42). This is done to avoid aliasing in the nonlinear evolution. The shading in the right panel shows the fluctuation range in time (5th-95th-percentile range) during the 1050-day window.

gives a better indication of the overall transport including the contribution due to eddies, and this is again direct at low altitude and reversed at high altitude in the region around $60^{\circ}$ latitude. The correspondence between the two panels of Fig. 13 is remarkable, but it should perhaps not be too surprising that the transport represented by the TEM streamfunction in the W2 and $\mathrm{ZS}$ cases is similar-that the zonal-mean flow in the latter case accomplishes some of what is done by the eddies in the former case-since this transport is ultimately driven by the external forcing, which is the same in both cases. We emphasize however that the form of this circulation is not a trivial consequence of the forcing: a Held-Hou-type solution with no overturning poleward of the zonal-wind maximum (despite the continuing horizontal temperature gradient) would have no streamfunction variation there at all, and so would not look like either of the panels of Fig. 13. The boundary of the direct circulation in both panels is close to the forcing tropopause at $200 \mathrm{~K}$; the adjacent $205-\mathrm{K}$ contour is shown dotted (as in Fig. 4). There is clearly scope for further work here to investigate the role of the vertical structure, in particular the static stability [which is highlighted by Held and Hoskins (1985)], in determining the detailed structure of the circulation.

At lower rotation rates, further details of the meanflow circulation near the pole are brought out by contour plots of the vertical pressure velocity $\omega$, in Fig. 14. The reversed overturning circulation noted at $\Omega=\Omega_{E} / 5$ is no longer evident in the $3 \mathrm{D}$ and $\mathrm{W} 2$ cases at $\Omega=\Omega_{E} / 100$ in Figs. 5d and 5h. In Fig. 14, the upward vertical wind on the poleward side of such a reversed circulation would show as blue $(\omega<0)$. For the W2 cases, a trace of it can still just be seen at $\Omega=\Omega_{E} / 50$ in Fig. 14 a, but not at $\Omega=\Omega_{E} / 100$ in Fig. $14 \mathrm{~b}$, and at $\Omega=\Omega_{E} / 200$ in Fig. $14 \mathrm{c}$ the downward Hadley circulation $(\omega>0$; red) extends all the way poleward. However, for the ZS cases (bottom row of Fig. 14), a reversed poleward circulation persists, and further structure is evident especially in Fig. 14e. The distinction between the W2 and ZS cases here may be due to the cross-pole circulation for W2 only, mentioned earlier. Note that since all of the features discussed here occur only within the troposphere (the upper part of all the panels is white), they are distinguished from the "deep" stratosphere-encompassing circulation observed by Caballero et al. (2008).

The dichotomy (6) and the thermal wind balance (11) were derived assuming the smallness of the meridional and vertical advection. The thermal wind equation is what relates the zonal wind and potential temperature profiles in the HH theory; it is used on both sides of the Hadley cell boundary, whichever set of matching conditions is adopted. We have seen in the present subsection that, unlike in the Hadley cell region, the overturning advection is not negligible and in fact these terms dominate the mean zonal momentum equation in the region poleward of the Hadley cell boundary. It is not then surprising that the continuity properties at the boundary, as well as the solution in the bulk of the poleward region, differ from the theoretical predictions.

It is possible that a new, simple, and physically motivated choice of flow field in the poleward region would lead to a new, simple theory in better agreement with the numerical results and perhaps with nicer continuity properties. The full specification of such a theory remains for further work, but we note that to take into account the temporal fluctuations successfully it may also be necessary to revisit the second matching condition, the integral constraint, which here corresponds to closure of the energy budget in steady state.

\section{Implications for modeling Venus}

Although we do not study Venus specifically in this paper, we wish to point out a couple of relevant 
(a) $\Omega_{E} / 5$ ( $m \leq 2$ only), $T 170$

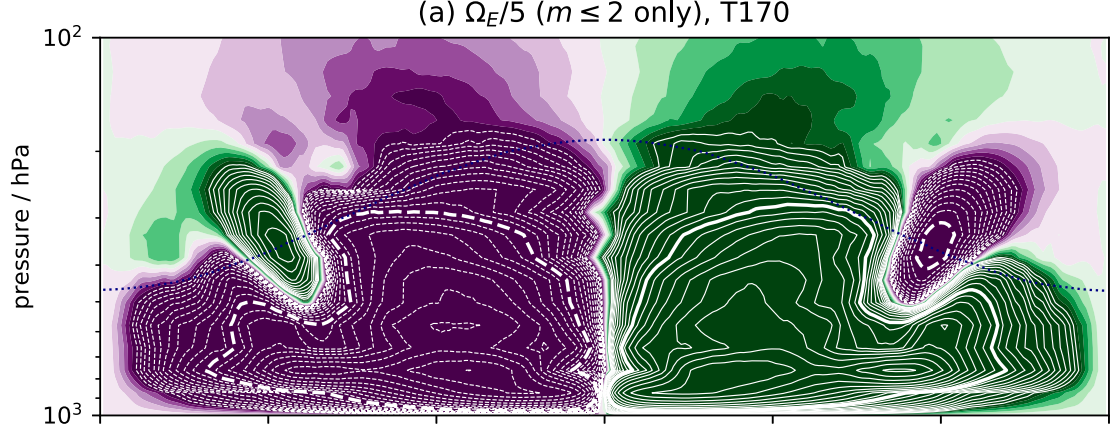

(b) $\Omega_{E} / 5$ ( $m=0$ only), T341

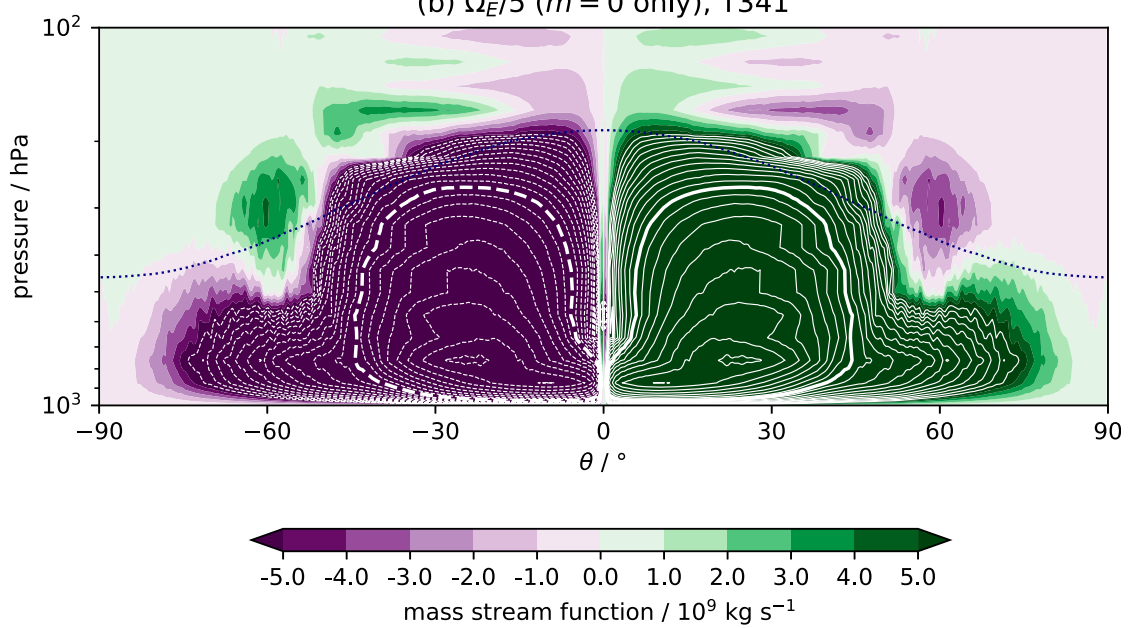

FIG. 13. The TEM mass streamfunction $\psi^{*}$ against latitude $\theta$ and model-level pressure (hPa), for $\Omega=\Omega_{E} / 5$ for cases (a) W2 and (b) ZS. In the ZS case, this is simply the usual Eulerian mean mass streamfunction $\psi$, and the shaded contour levels are chosen to highlight the low-value wings of the circulation in this case. The white contour lines indicate larger values, equally log spaced with 12 contour lines per decade. The thicker white lines mark the $\pm 5 \times 10^{10} \mathrm{~kg} \mathrm{~s}^{-1}$ level, which is the lowest level shown in Fig. 5 (negative contours are dashed). The thin dotted line shows the 205-K forcing contour, as in Fig. 4.

implications. First, in these simulations (which are all at Earth's surface pressure-that of Venus is two orders of magnitude higher), by the time $\Omega$ has decreased to $\Omega_{E} / 100$, which is still a faster rotation rate than Venus, the maximum zonal wind is already considerably lower than for Earth, even for the 3D simulations, whereas in reality Venus is observed to have faster zonal winds than Earth [e.g., in Venus Express data analyzed by Sánchez-Lavega et al. (2008)]. We find the same effect with higher surface pressures too (not included here). Some other mechanism is required in order to sustain the zonal winds on Venus, for example diurnal and semidiurnal thermal tides (e.g., Fels and Lindzen 1974), or effects arising from a better treatment of radiative forcing.

Our results may, however, contain clues to an explanation of Venus's strong polar vortex, and of its "cold collar" (e.g., Ando et al. 2016), which is a minimum in temperature (at a given altitude) at a latitude below the pole. In Fig. 4, at the highest rotation rates (left) the temperature decreases poleward, as does the forcing temperature, whereas at the lowest rotation rates (right) it is flattened across most of the planet and increases poleward aloft at very high latitude. Although our present results contain no strong examples of temperature first decreasing and then increasing poleward in the same case, it seems plausible that that may occur due partly to the type of transition seen here.

\section{Conclusions}

In this paper we have studied the zonally symmetric theory of HH and Hou (1984), and a variant with continuous zonal wind as its boundary matching condition, focusing mainly on the limit of low planetary rotation rate. We find that the original theory and this variant 


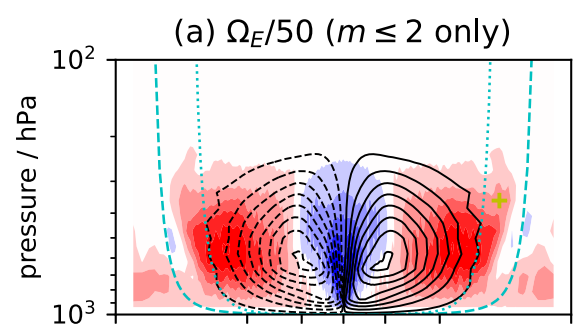

(d) $\Omega_{E} / 50$ ( $m=0$ only)

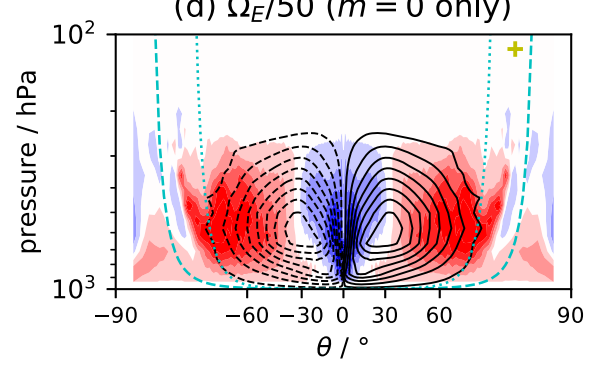

(b) $\Omega_{E} / 100(m \leq 2$ only)

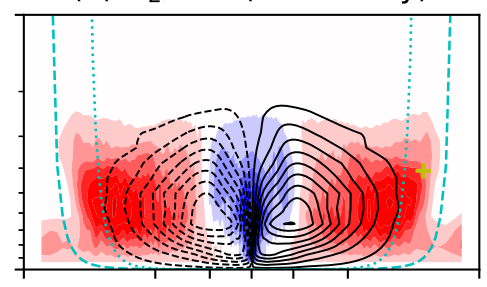

(e) $\Omega_{E} / 100$ ( $m=0$ only)

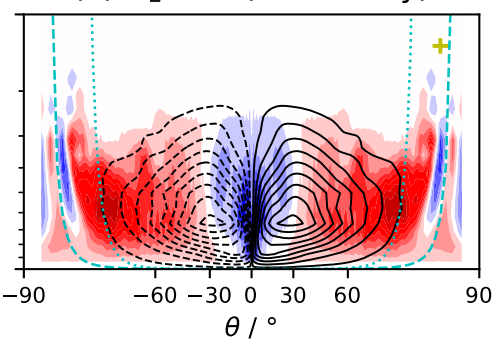

(c) $\Omega_{E} / 200$ ( $m \leq 2$ only)

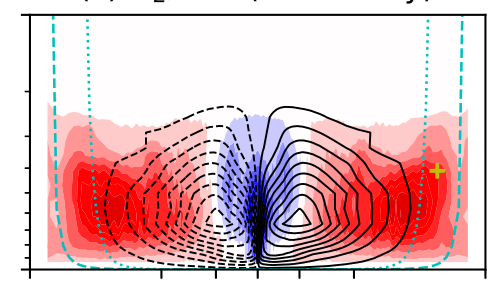

(f) $\Omega_{E} / 200$ ( $m=0$ only)

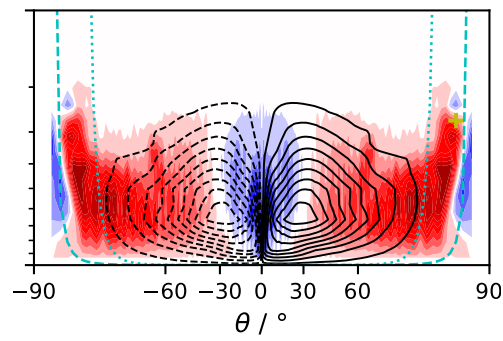

$\begin{array}{lllll}-0.2 & -0.1 & 0.0 & 0.1 & 0.2\end{array}$

$\omega / \mathrm{Pa} \mathrm{s} \mathrm{s}^{-1}$

FIG. 14. The vertical pressure velocity $\omega$ (color shading) against latitude $\theta$ and model-level pressure (hPa) for a different set of planetary rotation rates $\Omega$ (see panel titles). The yellow plus signs mark the maximum $u$, and contour lines indicate the mass streamfunction $\psi$, as in Fig. 5. The nonlinear latitude scale of Fig. 6 is used. These examples are all at T170 resolution for cases (a)-(c) W2 and (d)-(f) ZS.

have different scalings for the boundary colatitude and boundary zonal wind, as well as the different continuity properties. Simulations using a GCM are found to have smoother temperature profiles than either theory, and also feature an overturning circulation poleward of the point of maximum zonal wind, which is absent in both theories. It is the presence of this overturning circulation in the polar region (which occurs in both threedimensional and zonally symmetric simulations) that allows the zonal velocity profile to be smoother than the original theory, and removes the temperature discontinuities of the variant theory, without the need for viscous or diffusive smoothing. Resolved zonally symmetric simulations fall between the two sets of theoretical scalings, and have a faster polar zonal flow than both theories, consistent with a steepened polar temperature profile. While accepting that both theories are zonally symmetric by construction, they may still be informative for the three-dimensional case (Held and Hoskins 1985). The three-dimensional simulations fall closer to the predictions of the variant theory and the theoretical polar zonal flow, perhaps because allowed cross-polar flow removes the requirement to steepen the polar temperature profile to the same extent. Even in the zonally symmetric simulations, the maximum $u$ falls with falling $\Omega$ at rotation rates below $\Omega_{E} / 10$. This is easier to reconcile with the $u=0, \Omega=0$ case than the low- $\Omega$ limit of the original theory (which retains nonzero $u$ ), and indicates that something further would be required to sustain strong zonal winds at very low planetary rotation rates, such as are actually seen on Venus. The magnitude of the overturning circulation in the Hadley cell increases to a finite maximum as $\Omega \rightarrow 0$, and this is insensitive to whether zonally symmetric or not. The maximum overturning circulation is strong, in the sense that it drives the temperature profile close to a state of constant potential temperature.

More generally, our results show that the zonally symmetric theory is able to predict the qualitative behavior of the Hadley cell, and the associated zonal wind, as the planetary rotation rate is varied over quite a wide range (at least two orders of magnitude) below that of Earth. Both the original theory and its variant have quantitative shortcomings. Zonally symmetric numerical simulations (which is what the theories are constructed to describe) do not show a discontinuity in either zonal wind or temperature, at either low or high rotation rates, nor even a particularly fast variation of either quantity that might have been indicative of a discontinuity smoothed away by viscosity or diffusion. The reason for the smoothness seems to be that the region poleward of the edge of the Hadley cell is not in radiative equilibrium; rather, it has a nonzero circulation that enables the temperature to blend continuously with that in the Hadley cell region.

The three-dimensional simulations further differ from the theoretical predictions in two main ways. 
First, at high rotation rates, baroclinic eddies extract momentum (and heat) from the low-latitude Hadley cell, and the zonal wind does not quantitatively follow the angular-momentum-conserving profile, as is well known. Second, at low rotation rates, the three-dimensional simulations (even with the zonal structure limited to wavenumbers 1 and 2) produce superrotation.

Superrotation aside, the zonally symmetric theory is a better model of the three-dimensional circulation at low rotation rates than at high, because the lack of baroclinic eddies allows angular momentum to be better conserved. However, the reduction of the zonal wind at very low rotation rates is neither exactly predicted nor completely understood.

Acknowledgments. This work has been supported by the Leverhulme Trust and by NERC under Grant NE/M006123/1. We appreciate productive discussions with R. Geen, M. Jucker, F. Poulin, P. Maher, A. Paterson, J. Penn, and S. Thomson. We also thank I. Held and an anonymous referee for valuable suggestions. The SHTns library (Schaeffer 2013) was used to obtain the vorticity at the pole from its spectral representation.

\section{APPENDIX}

\section{Choice of $\boldsymbol{H}$ for Comparison with Numerical Simulations}

In section 6c we make the correspondence $\Delta_{H} g H \rightarrow$ $\Delta T_{y} R_{d}$ to go from the theory to the numerical model. In this appendix we obtain a more precise correspondence. We start from the following equation for thermal wind gradient balance in the numerical model:

$$
\frac{\partial}{\partial \ln p}\left(f u+\frac{u^{2} \tan \theta}{a}\right)=\frac{R_{d}}{a} \frac{\partial T}{\partial \theta} .
$$

Integrating from the surface, where $p=p_{s}$ and $u=0$, gives at pressure level $p$,

$$
f u+\frac{u^{2} \tan \theta}{a}=\frac{R_{d}}{a} \frac{\partial}{\partial \theta} \int_{p_{s}}^{p} T d(\ln p) .
$$

To obtain the equilibrium zonal wind (equivalent to $u_{E}$ in the main paper) we evaluate the right-hand side using $T=T_{\text {eq }}$, the Held-Suarez forcing (Held and Suarez 1994). Ignoring both the stratospheric cap temperature and the small term in $\ln \left(p / p_{0}\right)$, this is

$$
T_{\text {eq }}=\left(315 \mathrm{~K}-\Delta T_{y} \sin ^{2} \theta\right)\left(\frac{p}{p_{0}}\right)^{\kappa},
$$

where $p_{0}=1000 \mathrm{hPa}$ and $\kappa=2 / 7$. With $p_{s}=p_{0}$, we then obtain the same solution as (13), with the correspondence

$$
\Delta_{H} g H \rightarrow \frac{1}{\kappa}\left[1-\left(\frac{p}{p_{0}}\right)^{\kappa}\right] \Delta T_{y} R_{d} .
$$

Conveniently, the pressure at which this extra prefactor $(1 / \kappa)\left[1-\left(p / p_{0}\right)^{\kappa}\right]$ is equal to unity, $p / p_{0}=(5 / 7)^{7 / 2}=0.308$, is a good choice for a representative tropopause pressure (the actual level of maximum $u$ differs between runs, as indicated by the yellow plus signs in the figures), and the closest model level to this has been selected for Figs. 6 and 9. Justifying the simplifications leading to (A3), there is good agreement between the green curve and the dash-dotted curve in the top-left panel of Fig. 6, as expected from the correspondence between $T$ and $T_{\text {eq }}$ in Fig. 4 i.

The cyan curves in Figs. 5 and 14 are generated using (22) or (24) as appropriate, with $R$ dependent on $H$ according to (A4). The fact that the boundary of the angular-momentum-conserving region (in Fig. 5) approximately follows these curves, means that the particular choice of model level selected for comparison is not too critical. Solutions with similar boundaries were found by Fang and Tung (1996) to a slightly different problem, in which the vertical temperature profile was specified at a particular latitude to model convection at the intertropical convergence zone (ITCZ). This relative freedom to choose $H$ is likely connected with the lack of explicit vertical diffusion in our modeling. We intend to address the role of vertical diffusion further in later work.

\section{REFERENCES}

Adam, O., and N. Paldor, 2009: Global circulation in an axially symmetric shallow water model forced by equinoctial differential heating. J. Atmos. Sci., 66, 1418-1433, https://doi.org/ 10.1175/2008JAS2685.1.

Ando, H., N. Sugimoto, M. Takagi, H. Kashimura, T. Imamura, and Y. Matsuda, 2016: The puzzling Venusian polar atmospheric structure reproduced by a general circulation model. Nat. Commun., 7, 10398, https://doi.org/10.1038/ncomms10398.

Caballero, R., R. T. Pierrehumbert, and J. L. Mitchell, 2008: Axisymmetric, nearly inviscid circulations in non-condensing radiative-convective atmospheres. Quart. J. Roy. Meteor. Soc., 134, 1269-1285, https://doi.org/10.1002/qj.271.

Covey, C., E. J. Pitcher, and J. P. Brown, 1986: General circulation model simulations of superrotation in slowly rotating atmospheres: Implications for Venus. Icarus, 66, 380-396, https:// doi.org/10.1016/0019-1035(86)90166-1.

Fang, M., and K. K. Tung, 1994: Solution to the Charney problem of viscous symmetric circulation. J. Atmos. Sci., 51, 1261-1272, https:// doi.org/10.1175/1520-0469(1994)051<1261:STTCPO > 2.0.CO;2.

- and - 1996: A simple model of nonlinear Hadley circulation with an ITCZ: Analytic and numerical solutions. J. Atmos. Sci., 53, 1241-1261, https://doi.org/10.1175/15200469(1996)053<1241:ASMONH>2.0.CO;2. 
, and - , 1999: Time-dependent nonlinear Hadley circulation. J. Atmos. Sci., 56, 1797-1807, https://doi.org/10.1175/ 1520-0469(1999)056<1797:TDNHC $>2.0$. CO;2.

Fels, S. B., and R. S. Lindzen, 1974: The interaction of thermally excited gravity waves with mean flows. Geophys. Fluid Dyn., 6, 149-191, https://doi.org/10.1080/03091927409365793.

Ferrel, W., 1859: The motion of fluids and solids relative to the Earth's surface. Math. Mon., 1, 140-148, 210-216, 300-307, 366-373, 397-406.

Geen, R., F. H. Lambert, and G. K. Vallis, 2018: Regime change behavior during Asian monsoon onset. J. Climate, 31, 33273348, https://doi.org/10.1175/JCLI-D-17-0118.1.

Hadley, G., 1735: Concerning the cause of the general trade-winds. Philos. Trans. Roy. Soc., 29, 58-62.

Held, I. M., and A. Y. Hou, 1980: Nonlinear axially symmetric circulations in a nearly inviscid atmosphere. J. Atmos. Sci., 37, 515-533, https://doi.org/10.1175/1520-0469(1980)037<0515: NASCIA $>2.0 . \mathrm{CO} ; 2$.

, and B. J. Hoskins, 1985: Large-scale eddies and the general circulation of the troposphere. Advances in Geophysics, Vol. 28, Academic Press, 3-31, https://doi.org/10.1016/S00652687(08)60218-6.

, and M. J. Suarez, 1994: A proposal for the intercomparison of the dynamical cores of atmospheric general circulation models. Bull. Amer. Meteor. Soc., 75, 1825-1830, https://doi.org/10.1175/ 1520-0477(1994)075<1825:APFTIO>2.0.CO;2.

Hide, R., 1969: Dynamics of the atmospheres of the major planets with an appendix on the viscous boundary layer at the rigid bounding surface of an electrically-conducting rotating fluid in the presence of a magnetic field. J. Atmos. Sci., 26, 841-853, https://doi.org/10.1175/1520-0469(1969)026<0841: DOTAOT $>2.0 . \mathrm{CO} ; 2$.

Hou, A. Y., 1984: Axisymmetric circulations forced by heat and momentum sources: A simple model applicable to the Venus atmosphere. J. Atmos. Sci., 41, 3437-3455, https://doi.org/ 10.1175/1520-0469(1984)041<3437:ACFBHA > 2.0.CO;2.
Lindzen, R. S., and A. Y. Hou, 1988: Hadley circulations for zonally averaged heating centered off the equator. J. Atmos. Sci., $\mathbf{4 5}$, 2416-2427, https://doi.org/10.1175/1520-0469(1988)045<2416: $\mathrm{HCFZAH}>2.0 . \mathrm{CO} ; 2$.

Lorenz, E. N., 1967: The nature and the theory of the general circulation of the atmosphere. WMO Rep. 218, $161 \mathrm{pp}$.

Mitchell, J. L., and G. K. Vallis, 2010: The transition to superrotation in terrestrial atmospheres. J. Geophys. Res., 115, E12008, https://doi.org/10.1029/2010JE003587.

Plumb, R. A., and A. Y. Hou, 1992: The response of a zonally symmetric atmosphere to subtropical thermal forcing: Threshold behavior. J. Atmos. Sci., 49, 1790-1799, https://doi.org/10.1175/ 1520-0469(1992)049<1790:TROAZS > 2.0.CO;2.

Sánchez-Lavega, A., and Coauthors, 2008: Variable winds on Venus mapped in three dimensions. Geophys. Res. Lett., 35, L13204, https://doi.org/10.1029/2008GL033817.

Schaeffer, N., 2013: Efficient spherical harmonic transforms aimed at pseudospectral numerical simulations. Geochem. Geophys. Geosyst., 14, 751-758, https://doi.org/10.1002/ggge.20071.

Schneider, E. K., 1977: Axially symmetric steady-state models of the basic state for instability and climate studies. Part II. Nonlinear calculations. J. Atmos. Sci., 34, 280-296, https://doi.org/ 10.1175/1520-0469(1977)034<0280:ASSSMO > 2.0.CO;2.

Thomson, J., 1892: Bakerian lecture-On the grand currents of atmospheric circulation. Philos. Trans. Roy. Soc. London, 183A, 653-684, https://doi.org/10.1098/rsta.1892.0017.

Vallis, G. K., 2017: Atmospheric and Oceanic Fluid Dynamics. 2nd ed. Cambridge University Press, 964 pp.

— , and Coauthors, 2018: Isca, v1.0: A framework for the global modelling of the atmospheres of Earth and other planets at varying levels of complexity. Geosci. Model Dev., 11, 843-859, https://doi.org/10.5194/gmd-11-843-2018.

Zhang, G., and Z. Wang, 2013: Interannual variability of the Atlantic Hadley circulation in boreal summer and its impacts on tropical cyclone activity. J. Climate, 26, 8529-8544, https:// doi.org/10.1175/JCLI-D-12-00802.1. 